\title{
Dynamic Allocation of Downlink and Uplink Resource for Broadband Services in Fixed Wireless Networks
}

\author{
Kin K. Leung and Arty Srivastava \\ AT\&T Labs, Room 4-120 \\ 100 Schulz Drive \\ Red Bank, NJ 07701-7033 \\ Phone: (732)345-3153 \\ Fax: (732)345-3038 \\ Email: kkleung@research.att.com \\ December 3, 1997 \\ September 21, 1998 (Revised)
}

\begin{abstract}
We propose and analyze a resource allocation algorithm, referred to as the Enhanced Staggered Resource Allocation (ESRA) method, for downlink and uplink in fixed broadband wireless networks with directional antennas at base stations and terminals. The new method is based on the Staggered Resource Allocation (SRA) algorithm proposed in [FHLQS98] with consideration of reception quality at terminal locations [QC97]. Terminals are categorized into multiple classes, depending on their ability to tolerate concurrent packet transmissions. The bandwidth is divided into mini-frames allowing different degrees of concurrent transmissions, thus packets of different classes are sent in the corresponding mini-frames. The purpose of the ESRA method is to maximize concurrent transmissions up to an extent tolerable by the receiving terminals for throughput improvement, while avoiding major co-channel interference in the networks where the same frequency band is re-used in every sector of every cell.

For reasonable radio environment, practical antenna patterns and choices of system parameters, our performance analysis shows that the ESRA method provides $98.69 \%$ coverage, and yields a maximum downlink throughput of $36.10 \%$ per sector with success probability of one for packet transmission in terms of meeting a $15 \mathrm{~dB}$ threshold for signal-to-interference ratio (SIR). As for the uplink, using full power control, the throughput can reach $16.7 \%$ per sector with a packet loss probability of less than $5 \times 10^{-4}$. The ESRA performance is compared favorably to existing methods such as the CPDA scheme [BFZA96] and the SRA method [FHLQS98]. The high ESRA throughput translates into a very large network capacity. In addition to data services, the high quality of service reveals the applicability of the new method to support real-time traffic such as voice and video. Therefore, we believe that the new approach represents an important step forward in providing broadband, multi-media services in fixed wireless networks.
\end{abstract}




\section{INTRODUCTION}

As telecommuting and Internet access become very popular, the demand for broadband packet services is expected to grow tremendously. Customers are expecting high quality, reliability and easy access to high-speed communications from homes and small businesses. Broadband services are needed in the very near future for: a) accessing World Wide Web efficiently, b) providing data rates comparable to local-area networks for telecommuters to access their computers and data at the office, and c) providing multimedia services such as voice, image and video. Today, researchers and engineers are actively exploring the feasibility and conducting trials of several access technologies [B96] to provide the broadband services [197]. Promising technologies for broadband access include the asymmetrical digital subscriber loop (ADSL) [M96, HF97] and hybrid fiber-coax (HFC) [BMSS96], and wireless access networks [ZTL96].

In this paper, we consider the wireless approach for broadband, multimedia services. Specifically, we focus on a fixed broadband packet-switched network using time-division-multiple-access (TDMA) techniques with user data rates of $10 \mathrm{Mb} / \mathrm{s}$, link lengths typically less than 10 kilometers and operating frequency in the range of 1 to $5 \mathrm{GHz}$. The whole service area in the network is divided into cells. Each cell is further divided into multiple sectors, each of which is covered by a sector antenna co-located with a base station (BS) at the center of the cell. Because of the co-location, sector antennas are also called BS antennas. Terminals (users) use directional antennas mounted on the roof top and pointed to their respective BS antennas. The beamwidth of each $\mathrm{BS}$ antenna should be just wide enough to serve the whole sector, while a terminal antenna can have a smaller beamwidth to suppress interference. The ratios of front-to-back-lobe gain (abbreviated by FTB ratio below) for BS and terminal antennas are assumed to be finite. Time is slotted such that a packet can be transmitted in each slot. In addition, the downlink and uplink between terminals and BS can be provided either by time-division duplex (TDD) or frequencydivision duplex (FDD).

Among many design issues at the physical and link layer for the broadband wireless networks as discussed in [AEK96], a major challenge is spectral efficiency. If TDMA techniques are used to support a target data rate of $10 \mathrm{Mb} / \mathrm{s}$ for multimedia services, a bandwidth of several $\mathrm{MHz}$ is needed. Employing code-division-multiple-access (CDMA) techniques requires more bandwidth than TDMA does. Since radio spectrum is expensive, efficient strategies for re-using frequencies while adequately managing cochannel interference as a means to provide enough network capacity is critically important. In contrast to narrowband cellular networks where radio spectrum is divided into multiple channel sets, which are reused only in relatively distant cells (see e.g., [L89] and references cited in [KN96]), broadband wireless networks must re-use bandwidth very aggressively, ideally reusing the same frequency band in every cell. The need for reuse of a common radio spectrum in all cells has also been noted by [BFZA96] and 
[BZFTB96] for mobile broadband wireless networks. Having a shared communication channel with high data rate allows more efficient sharing of the limited bandwidth than in narrowband cellular networks.

In the context of packet-switched networks, time slots naturally become the bandwidth resources. We need to allocate time slots dynamically to various transmitters to send data packets such that a given signal-to-interference ratio (SIR) can be guaranteed at the intended receiver for successful reception. This results into the concept of dynamic resource allocation (DRA). The problem of time-slot assignment to achieve certain optimal performance while meeting a SIR requirement has been pointed out by [W96] to be NP-complete or hard [GJ79]. By using a central controller, [W96] and [KW97] proposed approaches to assigning time slots.

In our fixed wireless network, cell sectorization and directional antennas at fixed terminal locations are key components in reducing interference from neighboring sectors and cells. By exploiting this advantage, Fong et al. [FHLQS98] proposes the staggered resource allocation (SRA) method as a distributed DRA algorithm for the network where the same radio spectrum is used (shared) by each sector in every cell on a dynamic, time-division basis. With the use of directional antennas to suppress interference, the SRA method is particularly effective in avoiding both inter-cell and intra-cell interference.

Recently, Qiu and Chawla [QC97] make a novel observation: depending on terrain and fading, certain terminals (e.g., houses) due to their fixed locations, may be consistently unable to receive signal with satisfactory SIR while transmission for other terminals may always be successful. Thus, terminals at "good" and "poor" locations should be served according to different time-slot reuse patterns, which is called Time Slot Reuse Partitioning (TSRP) in [QC97]. The main idea is that many BS's can transmit simultaneously if the intended receiving terminals are located at good positions. On the contrary, when the receiving locations are poor, few BS's are scheduled to transmit at the same time so that a target SIR threshold can be met for successful reception at the receiving ends.

The specific implementation of TSRP in [QC97] divides the time frame (i.e., bandwidth) into a dedicated portion and a shared portion. At most one packet is transmitted among four neighboring cells during each time slot in the dedicated portion and up to three packets can be transmitted simultaneously in every cell in the shared portion. The purpose is to allow terminals at "good" and "poor" locations to use time slots in the dedicated and shared portion, respectively. Because of the bandwidth partitioning into the dedicated and shared portion, many terminal locations with moderate reception quality either may be over-protected when transmitting in the dedicated portion or may not receive the packets successfully when sent during the shared portion. It results into a potential waste of bandwidth, and has motivated this 
work.

Our purpose here is to enhance the SRA method in [FHLQS98] by considering the reception quality of terminals. The new method, to be referred to as the Enhanced Staggered Resource Allocation (ESRA) method, has the capability of avoiding major interference as the SRA method does, and makes use of the knowledge of the reception quality at terminals to improve throughput and maintain the success probability of one (or as close to one as practically possible) for downlink transmission. As discussed below, the ESRA also yields close to full downlink coverage for a set of typical radio and system parameters. As for the uplink, we show that the probability of having an SIR falling below a threshold, which is defined as the the packet loss probability, can be kept satisfactorily low when full power control is used. Furthermore, one can increase the uplink throughput up to an extent by concurrent transmissions among different sectors in each cell with an increase in the loss probability. For real-time applications such as voice and video, lost packets degrade the quality of service (QoS). As for non-real-time services, lost packets are subject to retransmission and long delay. Thus, the loss probability must and can be maintained by the ESRA method at a reasonable level for typical multimedia applications.

In the rest of the paper, we first give a brief description of the SRA method in Section 2. In Section 3, we present the ESRA method for the downlink in the fixed broadband wireless network. We analyze the downlink throughput, and discuss bandwidth sharing for the ESRA method in Section 4. Section 5 describes the uplink design and the power control algorithms. Using typical system parameters, Section 6 presents numerical results, discusses the performance characteristics of the ESRA method, and compares it to two existing methods. Finally, Section 7 is our conclusion.

\section{A BRIEF DESCRIPTION OF THE STAGGERED RESOURCE ALLOCATION (SRA) METHOD}

For illustration purposes, consider a regular, hexagonal cell layout in Figure 1. Each cell is divided into six sectors, each of which is served by a BS antenna with $60^{\circ}$ beamwidth, and terminal antennas can have a beamwidth smaller than $60^{\circ}$. In the SRA method, time slots are grouped into 6 subframes and sectors are labeled by 1 to 6 anti-clock-wise, as shown in Figure 1. The sector labeling patterns for adjacent cells differ by a $120^{\circ}$ rotation, thus creating a cluster of 3 cells whose patterns can be repeated across the entire system. Note that the time frame shown in the figure is applicable to both the downlink and uplink, which are provided by the TDD or FDD technique.

Each sector assigns time slots for transmitting packets to or from its terminals according to a special order shown in Figure 2. (In case a terminal needs to send packets to its BS, it is assumed that the BS is made aware of the need, perhaps via a separate dedicated channel or a contention channel.) For example, 
a sector with label 1 first schedules packets for transmission in time slots of subframe 1 (denoted by a) in the figure. If it has more traffic to send, it then uses subframe 4 (b), subframe 5 (c), etc. until subframe 6 (f). The reason for such an order is that, if interference due to concurrent packet transmission in the same cell can be tolerated, then after using all slots in the first subframe a, a sector should use the first subframe of the opposite sector in the same cell, in order to make the best use of the BS directional antennas. Following that, time slots in the first subframes for the sectors next to the opposite sector are used. To avoid interference due to overlapping antenna patterns of neighboring sectors, their first subframes are used as the last resort. For simplicity (while causing very minor throughput degradation), Figure 2 does not show the assignment from the left and right-hand side of the subframes as suggested in [FHLQS98]. As depicted in the figure, the assignment order for the next sector is "staggered" by a right rotation by one subframe based on the order for the previous sector. The assignment order, regardless of the associated sector, is generally referred to as the staggered order in the following.

It is easy to see from Figure 2 that if all sectors have traffic load of less than one-sixth of total channel capacity, all packets are transmitted in different time subframes, thus causing no interference within the same cell. Of course, as the traffic load increases, packets are transmitted simultaneously, thus increasing the level of interference. Nevertheless, the staggered order exploits the characteristics of directional antennas to allow multiple concurrent packet transmissions while reducing the intra-cell interference.

Besides managing intra-cell interference, the SRA method also helps avoid interference from major sources in the neighboring cells. This is particularly so when traffic load is low to moderate. To see this, let us consider the downlink for Sector 1 in the middle cell of Figure 1. Sector 2 in the bottom cell and Sector 3 in the upper cell are the major sources of interference. By examining the staggered order for Sector 1, 2 and 3, we find that they will not transmit simultaneously, so they will not interfere with each other provided that all of them have a traffic load of less than one-third of total channel capacity (i.e., using only subframes $\mathbf{a}$ and $\mathbf{b}$ for transmission). The same comment also applies to the uplink where Sector 2 and 5 of the bottom cell in the figure now become the major sources of interference. Due to the symmetry of the staggered order and cell layout, this same comment applies to each sector in every cell.

\section{THE ENHANCED STAGGERED RESOURCE ALLOCATION (ESRA) METHOD}

The Enhanced Staggered Resource Allocation (ESRA) method consists of the following components: terminal classification, selection of cell and sector, mini-frame structure, scheduling mechanism for packet transmission, selection of mini-frame sizes and power control. We shall focus on the downlink in the following and Section 4, while Section 5 discusses the method for uplink. 


\subsection{Terminal Classification}

The ESRA method uses the same sector labeling for the SRA method in Figure 1. The basic idea of the terminal classification in the ESRA method is to categorize terminals based on their ability to tolerate various degrees of concurrent packet transmissions according to the staggered order. Certainly such tolerance depends on the reception quality of the terminal locations, which in turn depends on the distance between the BS's and terminals, transmission power, antenna characteristics, terrain and fading. For the layout with six sectors per cell in Figure 1, there are six levels of concurrent transmission. Correspondingly, terminals are categorized into six classes, indexed by 1 to 6 .

As depicted in Figure 2, each time frame has 6 subframes, indexed by 1 to 6 . Let $J_{m}^{i}$ be the index of the $m^{\text {th }}$ subframe for use by sector $i$ in the staggered order. For example, $J_{1}^{3}=3, J_{2}^{3}=6, J_{3}^{3}=1, J_{4}^{3}=5$, $J_{5}^{3}=4$ and $J_{6}^{3}=2$ for sector 3 as it first uses slots in subframe 3, 6, 1, and so on, as shown in Figure 2. Further, for $c=1,2, \ldots, 6$, let $I_{c}(j) \subseteq\{1,2, \ldots, 6\}$ denote the set of sectors allowed to transmit in subframe $j$ when each sector can use only the first $c$ subframes in the staggered order for transmission (which results in $c$ concurrent packet transmissions in each cell). For instance, $I_{2}(1)=\{1,4\}$, $I_{3}(1)=\{1,4,3\}, I_{4}(3)=\{3,6,5,1\}$ and $I_{5}(3)=\{3,6,5,1,2\}$.

Assume that the system can activate one or a set of BS antennas to send a special signal such as a pilot tone for measurement purposes. The pilot tone is first activated by the BS antenna of the home sector where a terminal belongs. The received power of the pilot tone represents the signal strength at the terminal. Then, according to the possible concurrent transmissions allowed by the staggered order in different time subframes, a combination of BS antennas (including that of the home sector) in each cell are activated in a systematic way to send the pilot tone simultaneously. The total received power at the terminal is now the signal plus interference power. Thus, we can compute the SIR at the terminal for such different combinations. Terminal is categorized based on the highest degree of concurrent transmissions with its SIR exceeding a given threshold. To be precise, the following presents a classification procedure for a terminal located in sector $i$ :

a. $\quad$ Set $c=6$ and $k=1$.

b. Set $j=J_{k}^{i}$ (i.e., $j$ is the index of the $k^{\text {th }}$ subframe used by sector $i$ ). The system first instructs the BS antenna in the sector $i$ where the terminal belongs to transmit a special signal and the received signal strength is measured at the terminal location. Then, all sectors in $I_{c}(j)$ are arranged to transmit simultaneously and the received power (i.e., signal plus interference power) at the terminal is also measured. Thus, the SIR at the terminal when all sectors in $I_{c}(j)$ transmit can be obtained from these two measurements. If the SIR is less than a given threshold for satisfactory signal 
detection, continue with Step d. Otherwise, procceed with Step c.

c. If $k=c$, then the terminal is categorized as a class- $c$ terminal and the procedure is completed. (That is, it can sustain the interference with $c$ concurrent packet transmissions according to the staggered order.) Otherwise, increment $k$ by 1 and proceed with Step b (to check the SIR when transmitting in the next subframe).

d. If $c>1$, then decrement $c$ by 1 , set $k=1$ and proceed with Step b. Otherwise, the terminal cannot be served by the ESRA method (because the terminal is unable to meet the SIR threshold even when one packet is transmitted in each cell at a time) and the procedure stops.

As discussed in Section 6, for a typical radio environment, less than $1.5 \%$ of uniformly located terminals cannot be served by the ESRA method to meet a $15 \mathrm{~dB}$ SIR threshold. In these cases, terminal antennas with improved FTB ratio or sophisticated digital signal processing techniques to lower the SIR requirement may be used to ensure satisfactory reception at those locations.

In practice, the terminal classification can be done when installing the service at a terminal location. To consider possible changes of radio propagation conditions, the signal and interference power measurements should be taken and averaged over a sufficiently long period of time. Contrast to mobile networks, the propagation conditions in the fixed wireless network environment change relatively slowly. Thus, the measurement period may last for a few to more than ten minutes to ensure accurate characterization of reception quality at terminals. In addition, a "safety" margin can be included in the SIR threshold to guarantee satisfactory signal detection despite of fading and noise. With such steps, it is reasonable to expect all downlink transmissions can be successfully received with probability virtually equal to one, as will be assumed in the rest of this paper. Of course, to guard against changes of the propagation conditions in a slow time scale due to, for example, seasonal fluctuations, the classification of each terminal should be updated periodically by monitoring the reception quality through measurements and statistics collection.

One possible way to provide fast remedy to occasional inaccurate terminal classification or sudden change of propagation conditions such as an addition of man-made objects to the radio path, is to allow dynamic re-adjustment of terminal classification. For example, each lost packet is retransmitted up to a certain number of times and the associated terminal is re-classified from class $i$ to $i-1$, if the retransmission frequency for the terminal over a time period exceeds a certain threshold. If a significant number of terminals are re-classified, there may be a need to select new mini-frame sizes to meet the expected traffic load for all terminals, as discussed in Section 3.4. Nevertheless, with accurate terminal classification, such re-adjustment of terminal classification and mini-frame sizes is not expected to take 
place often.

\subsection{Selection of Cell and Sector}

It is well known that cell selection can improve quality of signal reception (e.g., see [Z97]). To take advantage of the macro-diversity in the ESRA method, each terminal selects its cell and sector, which may not necessarily be the closest one in distance, according to the shadow fading and the scheduling algorithm in use. Specifically, for each terminal, the ESRA method applies the terminal classification procedure presented above to determine its terminal class for several combinations of sectors and cells in the vicinity of the terminal. Then, the terminal chooses its home sector and cell that gives the terminal class with the largest index (i.e., that can tolerate the highest degree of concurrent transmission). If multiple combinations of cells and sectors yield the same terminal class, then the one with the highest SIR among them is chosen.

Now, with the terminal classification and selection of cell and sector, packets for a class- $c$ terminal can be received successfully as far as meeting the required SIR is concerned, if each sector uses the first $c$ subframes in the staggered order (which yields $c$ concurrent packet transmissions in each cell). For this reason, as discussed in the following, we can modify the frame structure in Figure 1 so that packets for each terminal class can now be transmitted simultaneously up to the maximum tolerable degree of concurrent transmissions in order to improve the throughput without degrading the success probability of packet reception.

\subsection{Frame Structure and Scheduling Mechanism}

Each time frame in the ESRA method consists of six subframes, indexed by 1 to 6 in Figure 3 . Each subframe is further divided into six mini-frames, which are also labeled from 1 to 6 . Each miniframe with the same label consists of multiple but fixed number of time slots in each subframe. The sizes of mini-frames are chosen to match the expected traffic demand of the terminal classes; see details in the next section.

Each sector uses the subframes according to the staggered order, given by $\mathbf{a}, \mathbf{b}, \mathbf{c}, \mathbf{d}, \mathbf{e}$, and $\mathbf{f}$ in the figure (where a and $\mathbf{f}$ indicates the first and last subframe to be used, respectively). However, it is important to note that time slots of only those mini-frames marked with dashed lines are available to the corresponding sector indicated on the left-most side of the figure. Clearly, varying from subframe to subframe, each sector is allowed to schedule packet transmission in one or more mini-frames in some subframes, but not in others. For instance, sector 2 can use all mini-frames in subframe 2, but it can schedule transmission only in mini-frame 5 and 6 in subframe 3; other mini-frames in subframe 3 are 
unavailable to sector 2 . It is also noteworthy that there are different degrees of concurrent packet transmission in various mini-frames. For $c=1,2, \ldots, 6$, as many as $c$ packets are transmitted simultaneously in each cell during mini-frame $c$ in each subframe. On one extreme, only one packet is transmitted in each cell during mini-frame 1, while on the other extreme, up to six packets are sent during mini-frame 6. The main idea here is that various mini-frames allows different degrees of concurrent packet transmissions. Thus, the mini-frame structure is compatible with the terminal classification so that packets for class- $c$ terminals transmitted in mini-frame $c$ will be successfully received as verified in the classification procedure. In fact, as to be discussed further later, packet transmission for a class- $k$ terminal in mini-frame $c$ with $c<k$ (referred to as upgraded sharing) will also be successfully received. The upgraded sharing helps avoid loss of "trunking efficiency" by partitioning subframes into mini-frames.

Based on the compatibility between the mini-frame structure and terminal classes, each sector schedules packet transmissions in the ascending order of terminal class indices. That is, traffic for class 1 and 6 terminals are arranged for transmission first and last, respectively. Time slots in mini-frames with small indices, starting from those mini-frames in the first subframe of the staggered order, are used first, thus enabling upgraded sharing as much as possible in case mini-frames are not fully utilized by traffic of their associated terminal classes. To be precise, the following procedure is invoked for each time frame by each sector in every cell to assign available time slots in the frame to pending packets for transmission. Once a packet is scheduled for transmission in a time slot, the slot becomes unavailable to other packets.

a. Set $c=1$ and $i=c$.

b. The sector schedules pending packets of terminal class $i$ for transmission in the available time slots of mini-frame $c$, starting from the first subframe in the staggered order (denoted by a to $\mathrm{f}$ ) and according to the availability of mini-frames in the subframes to the sector as shown in Figure 3. The scheduling continues until either i) all available time slots in mini-frame $c$ have been assigned, or ii) all pending packets for the terminal class have been scheduled for transmission. If condition i) occurs, then proceed with Step c; otherwise, proceed with Step d.

c. If $c \leq 5$, then increase $c$ by 1 , set $i=c$ and proceed with Step b. Otherwise, the procedure stops as all available time slots in the time frame have been assigned.

d. If $i<6$, increase $i$ by 1 and proceed with Step b (to schedule transmission of packets for the next terminal class in mini-frame $c$ ). Otherwise, the procedure stops as all pending packets have been scheduled for transmission. 
It is worth noting that as Step b and d do, as long as time slots are available, packets are transmitted by the upgraded sharing to further enhance the SIR at the receiving ends.

\subsection{Selection of Mini-Frame Sizes}

The mini-frame structure can be viewed as a means to divide the bandwidth into multiple "channels" that allow different degrees of concurrent packet transmissions tolerable in terms of SIR by various terminals. So, in order to maximize the system throughput, the sizes of mini-frames should be chosen to match the traffic load from the respective terminal classes.

Without loss of generality, consider that terminals of all classes have identical traffic load. Let $\alpha_{i}$ be the fraction of class- $i$ terminals (relative to the total number of terminals served by the ESRA method) in the whole network for $i=1$ to 6 . Further, let $N_{t}$ be the "target" number of time slots in each subframe, which is determined by considering packet delay requirements, scheduling overhead and so on. In addition, let mini-frame $i$ in each subframe have $n_{i}$ time slots. Observe that each sector can use miniframe $i$ in $i$ different subframes (see Figure 3). Therefore, to handle the uniform traffic load among terminals,

$$
n_{i}=\beta \frac{\alpha_{i}}{i}
$$

where $\beta$ is a proportionality constant and the rounding of integer is ignored at the present moment. Since

$$
\begin{aligned}
& \sum_{i} n_{i}=N_{t}, \beta=\frac{N_{t}}{\sum_{j} \alpha_{j} / j} \text {. Substitute this into (1) yields } \\
& \qquad n_{i}=\left\lceil\frac{N_{t} \alpha_{i} / i}{\sum_{j} \alpha_{j} / j}\right\rfloor
\end{aligned}
$$

where $[x]$ denotes the integer closest to $x$. With these mini-frame sizes, each subframe has $N=\sum_{i} n_{i}$ time slots and the frame size is $K N$ where $K$ is the number of sectors in each cell (which equals 6 for the setting under consideration).

\section{DOWNLINK PERFORMANCE OF THE ESRA METHOD}

In this section, we analyze the maximum packet throughput for the downlink in each sector, and discuss efficient sharing of mini-frames among terminal classes for the ESRA method. 


\subsection{Maximum Packet Throughput Per Sector}

To begin, it is important to recall that by the terminal classification, packet transmissions for each terminal class in its respective mini-frame will be successful. That is, the success probability of packet transmission is one as far as meeting a specific SIR threshold is concerned.

To analyze the packet throughput for the ESRA method, we continue to assume that terminals of all classes have identical traffic load. Further, assume that there are always packets pending for transmission. Based on the size of each mini-frame $i$ in (2), the maximum throughput for class- $i$ terminals is $i n_{i} / K N$ packets per time slot in each sector. This is so because: 1) each sector can transmit during mini-frame $i$ in $i$ different subframes of each frame, and 2) each packet transmission for class- $i$ terminals in mini-frame $i$ will be successful by the definition of terminal classification. Thus, using (2), the maximum throughput in each sector for all terminal classes is

$$
\gamma_{m}=\sum_{i} \frac{i n_{i}}{K N}=\sum_{i} \frac{i}{K N}\left\lfloor\frac{N_{t} \alpha_{i} / i}{\sum_{j} \alpha_{j} / j}\right\rfloor
$$

Ignoring the rounding for integer in (3), and applying the facts that $\sum_{i} \alpha_{i}=1$ and $N_{t} \approx N$, we obtain the maximum downlink throughput per sector

$$
\gamma_{m} \approx \frac{1}{K \sum_{j} \alpha_{j} / j}
$$

Since the success probability of downlink transmission in the ESRA method is one, its throughput is only limited by the availability of pending packets associated with each terminal class for a given mini-frame structure. As a desirable consequence, once the maximum throughput is reached for sufficient traffic, further increase of traffic load will not cause any throughput degradation.

\subsection{Sharing Mini-Frames Among Terminal Classes}

At the first appearance, the bandwidth partition into mini-frames in the ESRA method may lead to loss of "trunking efficiency." However, packets for class- $k$ terminals can be transmitted successfully during any mini-frame $c$ available to the associated sector with $c \leq k$. When such a packet is sent in miniframe $c<k$, the SIR can actually be improved at the receiving ends. Thus such sharing of mini-frames is referred to as upgraded sharing. The SIR improvement can be seen readily from the terminal classification procedure because when $c<k$, the set of all interfering transmitters in a mini-frame $c$ is a subset of all interferers for a class- $k$ terminal. A detailed analysis of the SIR improvement is omitted here 
for brevity.

In contrast, one can show that transmitting class- $k$ packets during available time slots in mini-frame $c>k$, to be called downgraded sharing, cannot guarantee the satisfactory SIR. That is, the downgraded sharing does not yield successful packet transmission with probability one. For this reason, the scheduling algorithm in Section 3.3 does not include such sharing. Nevertheless, the downgraded sharing can still be applied to packets without tight delay requirements. This is particularly so if the BS can schedule class- $k$ packets for transmission in time slots of mini-frame $c>k$ when the traffic load is sufficiently low that the degree of concurrent packet transmissions in those slots can be kept to be no more than $k$. It is important to note that packet transmissions by the downgraded sharing have no impact on the original transmissions or those by the upgraded sharing because the downgraded sharing does not increase the degree of concurrent transmissions, thus keeping the interference level unchanged. In the

worst case, if packets are not received successfully in the first attempt by the downgraded sharing, they can be re-transmitted in their corresponding or upgraded mini-frames. Due to the extra delay in retransmissions, the downgraded sharing will be mainly applicable to non-real-time applications.

\section{UPLINK DESIGN IN THE ESRA METHOD}

The sector labeling, selection of cell and sector, frame structure and scheduling mechanism, and selection of mini-frame size discussed in Section 3 also apply to the uplink. It is assumed that terminals with packets ready for transmission first notify their associated BS's, perhaps via a separate dedicated channel or a contention channel. The ESRA method focuses on the scheduled packet transmissions on the uplink; see e.g., [SK98] for a survey of contention-based MAC protocols for broadband wireless networks.

\subsection{No Guarantee for Uplink SIR}

We remark that due to the terminal classification procedure, the downlink SIR performance for any terminal is guaranteed in the ESRA method because all BS antennas associated with the terminal class are activated to transmit in the procedure, thus causing the maximum amount of interference. On the other hand, there are many more terminals than the number of sectors in the system, the transmitter locations for uplink change in every time slot, according to the scheduling mechanism. It is found that even assuming all terminals belong to class one (i.e., one terminal transmits in each cell in a time slot), the uplink SIR for a terminal can be unsatisfactorily low in a typical radio environment, when the strongest interfering terminal in each of the sectors associated with the terminal class in neighboring cells is transmitting. Thus, the uplink SIR performance cannot be guaranteed. Instead, our design goal is to 
achieve an adequately low probability for having the uplink SIR for any terminal below a given threshold. This probability is referred to as the packet loss probability (which equals one minus the success probability of packet transmission discussed above). Indeed, the packet loss probability can be kept satisfactorily low with two concurrent packet transmissions in each cell for a typical radio environment. Depending on applications, real-time traffic such as voice and video will require a low probability, while data can tolerate a high value (e.g., perhaps up to of 10\%) as erroneous data transmissions can be recovered by ARQ techniques. Thus, in contrast with the terminal classification for the downlink, we have traffic classification for the uplink in the ESRA method. That is, traffic of various applications is transmitted in different mini-frames on the uplink, depending on their packet loss probability requirements. Naturally, in accordance with the expected traffic load for these applications, the miniframe sizes can be chosen. As discussed in Section 6, uplink traffic can be categorized into class 1 and 2, where class 1 can provide lower packet loss probability suitable for real-time traffic.

\subsection{Uplink Power Control}

Another key distinction between uplink and downlink in the ERSA method is that power control ought to be used for uplink transmissions, especially when concurrent transmissions are allowed within the same cell. Dynamic power control in cellular networks has been widely studied and practiced to manage interference; see e.g., [Z92a], [W93] and [HWJ97]. Note that iterative power control algorithms such as those in [Z92b] and [FM93] are applicable only to voice calls because of their relatively long holding time. However, they are inapplicable to data packets due to the burstiness and irregular transmission schedule in the networks under consideration. Furthermore, it is also difficult to apply SIRbased power control techniques to packet transmissions on the uplink. This is so because as pointed out above, transmitter locations keep changing, thus causing difficulty in estimating the SIR and errors in adjusting the transmission power. Thus, signal-based power control [W93] appears to be the only feasible candidate.

Recently, [HWJ97] proves that the square-root power control (SPC) (i.e., to compensate half of the path loss and shadow fading in $\mathrm{dB}$ ) maximizes the SIR between two pairs of mobiles and BS's that interfere with each other, with a hope of increasing the frequency reuse in cellular networks. For the ESRA method, we prove in the following that for a single-cell setting with a reasonable FTB ratio, the full power control (FPC) that fully compensates the path loss and shadow fading maximizes the probability of having SIR exceeding a given threshold (or equivalently, minimizes the packet loss probability). Our purpose in using the FPC is to achieve a low packet loss probability for the ESRA method. 
To show the optimality of the FPC for our networks, let us consider a single cell with $M$ terminals in $M$ different sectors (indexed by 1 to $M$ ) scheduled for transmission in a time slot. We assume that the BS antenna serving each sector has a simplified radiation pattern with a front and back lobe where the latter also includes the side lobe. Let $\tau$ denote the FTB ratio for the antenna. For any signal-based power control scheme A, let $S_{i}^{A}$ be the signal strength received at the intended BS antenna of sector $i$ from its transmitting terminal in a time slot. Note that since different terminals in the same sector are scheduled to transmit in different time slots, $S_{i}^{A}$ can be viewed as a random variable, depending on the path loss and shadow fading of the transmitting terminal, and the power control scheme in use. As sectors schedule independently, $S_{i}^{A}$, s are independent among all sectors $i$. Furthermore, by assuming terminals in one sector have identical statistics for path loss, shadowing and traffic load as those in all other sectors, $S_{i}^{A}$, s are independent identically distributed (i.i.d.) for all $i$. Due to signal suppression by the directional antenna, $\tau S_{i}$ is the interference power for transmissions in other sectors. Let $\Phi$ be the objective SIR threshold for successful reception.

Theorem. If $\Phi \tau(M-1) \leq 1$, among all signal-based power control $A$, the full power control (FPC) maximizes the minimum SIR among all packet transmissions in the multi-sector, single-cell setting. That is, the FPC maximizes

$$
\left.\min _{i} \operatorname{Pr} \mid \frac{S_{i}^{A}}{\sum_{j \neq i} \tau \overline{S_{j}^{A}}} \geq \Phi\right\rfloor
$$

over all signal-based power control schemes.

Proof. Due to symmetry of identical statistics for path loss, shadowing and traffic load for various terminals in all sectors, maximizing (5) requires that the probabilities for all sectors $i$ be identical. So, let us consider the probability only for an arbitrary sector $i$. Without loss of generality, for a signal-based power control scheme $A$ other than the FPC, let $S_{i}^{A}$ equal $x_{j}$ with a probability $\pi_{j}>0$ for $j=1,2, \ldots, n$ where $\sum_{j=1}^{n} \pi_{j}=1$ and $0<x_{1}<x_{2}<\cdots<x_{n}$. Note that $n \geq 2$ because of the non-perfect power control that always leaves certain amount of path loss and fading uncompensated for or over-compensates for them. Since $S_{i}^{A}$ has the smallest value of $x_{1}$ and $\Phi \tau(M-1) \leq 1$,

$$
\left.\operatorname{Pr} \mid S_{i}^{A} \geq \Phi \tau \sum_{j \neq i} S_{j}^{A}\right\rceil<\operatorname{Pr}\left[S_{i}^{A} \geq \Phi \tau(M-1) x_{1}\right]=1
$$

In contrast, by definition of the FPC, $S_{i}^{F P C}$ is a constant, say $c$. Since $\Phi \tau(M-1) \leq 1$, we have 


$$
\left.\operatorname{Pr} \mid S_{i}^{F P C} \geq \Phi \tau \sum_{j \neq i} S_{j}^{F P C}\right\rceil=\operatorname{Pr}[c \geq \Phi \tau(M-1) c]=1 .
$$

Comparing (6) and (7) thus proves that the FPC maximizes (5) under the condition $\Phi \tau(M-1) \leq 1$.

There are two ways to interpret the physical meaning of the condition, $\Phi \tau(M-1) \leq 1$. First, when all transmitting terminals in various sectors have identical combined path loss and fading, the inequality reveals that without any power control, the concurrent transmissions will meet the SIR threshold. Alternatively, when the FPC scheme is used, the condition guarantees the SIR exceed the threshold $\Phi$ as the interference can be adequately suppressed by the directional antenna. The latter is particularly helpful in determining the degree of concurrent uplink transmissions, $M$, by measurements in the field.

It is clear that the Theorem does not fullly address the power control issue for our multi-cell networks. In particular, the optimal power control (in terms of maximizing the SIR for a given packet loss probability) that covers both the intra- and inter-cell interference is yet to be found. However, since the ESRA method allows concurrent uplink transmissions within the same cell and since intra-cell interference can be significant because of short distance between the interferers and the BS, we conjecture that, for the range of SIR threshold of 10 to $15 \mathrm{~dB}$ for typical modulation and equalization (e.g., QPSK and DFE), the optimal power control in the multi-sector, multi-cell networks will be much closer to the FPC than to the SPC. Our numerical results in the following also support such belief.

\section{NUMERICAL RESULTS AND DISCUSSION}

The purpose of this section is to illustrate the performance of the ESRA method for typical radio and antenna parameters. We use an OPNET $\dagger$ simulation model to obtain the fraction of terminals of different classes. Based on the fractions and the assumption of uniform traffic among terminal classes, we apply the analysis result in Section 4 to compute the downlink throughput. We also show the uplink SIR distributions for various power control schemes. In addition, the ESRA performance is compared with that of the CDPA and SRA method.

$\dagger$ The OPNET simulation package is developed by Mil 3, Inc. 


\subsection{Model and System Parameters}

A two-tier, hexagonal-cell layout with a total of 19 cells is simulated in the model. That is, an outer tier of 12 cells is added to the configuration in Figure 1. Each cell is divided into 6 sectors, each of which is served by a BS antenna co-located at the center of the cell. Unless specified otherwise, the beamwidth (where the signal strength drops by $3 \mathrm{~dB}$ ) of each BS and terminal antenna is $50^{\circ}$ and $30^{\circ}$, respectively, while each terminal antenna points directly to its BS antenna. Practical antenna patterns are used in this study. Figure 4 gives a typical pattern for the front lobe of an antenna. Although the back/side lobe is not shown, signal arriving at the back/side lobe is attenuated according to the FTB ratio. Due to the overlapping of antenna patterns, it is likely for certain terminals, especially those located at the sector boundary, to receive a significant amount of interference from the neighboring sectors.

Each radio path between a transmitter and a receiver for both downlink and uplink is characterized by a path-loss model with an exponent of 4 [R96, p.102] and lognormal shadow fading. For the downlink, since there is only one radio path between all BS antennas in the same cell (which are co-located) and any terminal in the cell, the intended signal and interference experience the same lognormal fading and path loss. However, the fading from BS antennas at other cells are assumed to be different and independent. Unless stated otherwise, we assume the typical FTB ratios for BS and terminal antennas (denoted by B and $\mathrm{T}$ ) of 25 and $15 \mathrm{~dB}$, respectively. The standard deviation for the shadow fading is $8 \mathrm{~dB}$. Furthermore, with standard modulation and equalization schemes (e.g., QPSK and DFE), the SIR threshold for satisfactory detection probably lies between 10 to $15 \mathrm{~dB}$. Thus, we choose a SIR threshold of $15 \mathrm{~dB}$ in this study. For each packet transmission, if the SIR at the intended receiver exceeds the threshold, the packet is considered to be successfully received.

To avoid non-uniform performance in the outer layers of cells, only the statistics in the middle cell are collected and reported here. More precisely, our downlink results are obtained for about 1,000 terminals uniformly placed throughout sector 1 in the cell located at the center of the layout. To study the uplink performance, 20 terminals are randomly placed in each sector in the 19-cell layout. As for power control, we assume no power control for the downlink, while various power control schemes are considered for the uplink.

\subsection{Fractions of Terminals in Various Classes for the Downlink}

Following the classification method in Section 3, Table 1 presents the fraction of terminals in various classes for the SIR threshold of $15 \mathrm{~dB}$. Results with or without BS and sector selection (to be called BS selection in short) are included. The sum of the fractions for all classes gives the total fraction of terminals that can be served by the ESRA method and that is referred to as coverage. Since the ESRA 
method can eliminate intra-cell interference entirely by allowing only one packet transmission in each cell at a time, one can expect that the coverage is determined mainly by inter-cell interference and fading. On the other hand, the maximum downlink throughput strongly depends on the fractions of terminals in various classes. That is, the higher degree of concurrent transmissions is tolerable to terminals, the higher the throughput it yields.

Without BS selection, the coverage is $85.94 \%$ in Table 1; that is, the remaining $14.06 \%$ of terminals cannot be served because they cannot meet the SIR threshold due to strong inter-cell interference, even when there is only one packet transmission in each cell at a time. In contrast, the coverage sharply increases to $98.64 \%$ by BS selection because of the macro-diversity advantage and such coverage is adequate in practice. (In fact, our results also show that the coverage can be further improved when the FTB ratio for terminal antennas is higher than $15 \mathrm{~dB}$.) In addition, BS selection tends to make terminals more tolerant of a high degree of concurrent packet transmissions when compared with the case without BS selection.

It is also worth noting that the table reveals a vast majority of the terminals in class 2 , but rather a small fraction in class 3 to 5 . This is so because the staggered order is particularly good at avoiding both intra- and inter-cell interference when each sector transmits in the first two subframes in the order. However, for higher degrees of concurrent transmissions, the amount of intra-cell interference increases due to the overlapping antenna patterns between adjacent sectors in the same cell. Nevertheless, there are $16.98 \%$ of class- 6 terminals, which are likely to be located close to the BS with favorable fading and not affected by the BS antenna patterns from the adjacent sectors, thus being able to satisfy the SIR threshold.

\subsection{Downlink Impacts due to Antenna Beamwidth and FTB Ratios}

In Figure 5 to 7, we portray how the antenna beamwidth and FTB ratios impact the performance of the ESRA method. Figure 5 shows the impacts of BS antenna beamwidth on the maximum downlink throughput and coverage. As indicated by the upper curve in the figure, the coverage is quite insensitive to the beamwidth. However, the maximum throughput (depicted by the lower curve) can be improved if we reduce the $3 \mathrm{~dB}$ beamwidth from $60^{\circ}$ to a smaller value. This is because a narrower beamwidth yields a reduction of interference to neighboring cells and sectors. Of course, too small a beamwidth may also decrease the signal strength to an unacceptable level when compared with thermal noise, which is not considered in this study. Nevertheless, a beamwidth of about $50^{\circ}$ for BS antenna will be appropriate to serve a $60^{\circ}$ sector.

In fact, we also vary the beamwidth of the terminal antenna from $10^{\circ}$ to $40^{\circ}$, while keeping the $\mathrm{BS}$ antenna beamwidth at $50^{\circ}$ and other system parameters unchanged. It is found that the ESRA 
performance is very insensitive to the range of terminal antenna beamwidth. This is intuitively correct because as long as the beamwidth is less than $60^{\circ}$, each terminal antenna, while pointing directly to its own BS, faces toward the front lobe of the BS antenna of at most one sector in the first-tier neighboring cells of the layout under consideration, which contributes most of the inter-cell interference for the terminal.

Figure 6 portrays the downlink performance impacts due to the FTB ratio of BS antenna. As explained above, the coverage mainly depends on inter-cell interference, especially from those BS antennas with front-lobe facing the front-lobe of the terminal antennas. As a result, the coverage is relatively insensitive to the FTB ratio of BS antenna as shown in the figure. On the other hand, as the FTB ratio increases, interference decreases, which in turn allows a high degree of concurrent transmissions, thus improving the maximum packet throughput. However, for the given parameter setting, when the FTB ratio reaches $25 \mathrm{~dB}$, further increase of the ratio yields only marginal throughput improvement. This is so because other parameters such as the FTB ratio for terminal antennas and the SIR threshold become dominating factors in determining the throughput.

In contrast, Figure 7 shows that both downlink coverage and throughput strongly depend on the FTB ratio for terminal antennas. Generally, when the ratio is high, inter-cell interference can be suppressed sufficiently so that almost all terminals meet the SIR threshold (with one packet transmission per cell in the worst situation) and terminals can tolerate a high degree of concurrent transmissions. As a result, both coverage and throughput improve as the terminal FTB ratio improves.

\subsection{Comparison of Downlink Performance With CDPA and SRA Method}

We compare the ESRA downlink performance with the CPDA [BZFTB96, BFZA96] and SRA [FHLQS98] method. These methods are chosen for comparison because both of them are proposed for wireless packet access and furthermore the ESRA method is derived from the SRA method.

Using simulation techniques, Figure 8 and 9 compare the throughput and packet success probability between the CDPA and the ESRA method, respectively, as a function of normalized aggregated load (i.e., new plus retransmissions). To adopt the CDPA protocol to the setting with directional antennas as described in Section 6.1, we add a parameter (denoted by $C$ in the figures) to control the number of downlink concurrent transmissions in each cell. When $C=6$, all six BS (sectoral) antennas in each cell can transmit simultaneously. However, for $C<6, C$ sectors in each cell are randomly selected for possible transmission in a time slot. Whether such a selected antenna actually transmits or not depends on its traffic load condition. The receiving terminal is randomly selected from those in each sector. 
To have a fair comparison, we do not consider power control and forward-error-control code for the CDPA as [BZFTB96] does. In addition, terminals using the CDPA scheme select their base station and sector based on the strongest signal strength. Using the system parameters discussed in Section 6.1 and the SIR detection threshold of $15 \mathrm{~dB}$, Figure 8 shows that the CDPA scheme yields higher throughput for higher $C$ values at low traffic conditions (where the throughput is defined as the expected number of packet with SIR exceeding the $15 \mathrm{~dB}$ threshold at the receiving terminal in each sector per time slot). As traffic increases, high $C$ values cause too much interference, thus reducing the channel throughput. As for the ESRA method, when traffic load is below $36.1 \%$ (as indicated in Table 1), all packet transmission will be successful as its SIR is higher than the $15 \mathrm{~dB}$ threshold as verified by the procedure for terminal classification. When traffic load goes beyond $36.1 \%$, all time slots in various mini-frames and subframes available to associated sectors are fully used for transmission. Since the SIR for all these transmissions exceeds the $15 \mathrm{~dB}$ threshold, the throughput is maintained at $36.1 \%$ despite further increase of traffic load. It is worthnoting that the ESRA performs much better than the CDPA for all $C$ values because the former makes use of the staggered order to avoid major interference, and schedules transmission according to the terminal classification for maintaining the SIR performance.

Figure 9 shows that the packet success probability for the CDPA method deteriorates rapidly as $C$ increases at high traffic load conditions. In contrast, the packet success probability for the ESRA method remains one as far as meeting the $15 \mathrm{~dB}$ SIR threshold is concerned.

Similarly, figure 10 and 11 compare the performance of ESRA and SRA method. As discussed in [FHLQS98], a control mechanism can be applied in the SRA method to limit packet transmissions only in the first few subframes of the staggered order for each sector. The number of such subframes is denoted by $C$ in the figures. Note that $C$ also represents the number of concurrent transmissions in each cell in the SRA method. Figure 10 presents the throughput comparison between the SRA and ERSA method. In most of the cases, the ESRA method gives higher throughput than the SRA method. An exception is that the SRA method with $C=3$ and 4 yields higher throughput than the ERSA method when traffic load exceeds about 70 or $80 \%$. However, the tradeoff is showed in Figure 11, which indicates that the packet success probability drops significantly for the SRA method, especially at high load condition. Even worse is that the packet loss in the SRA method does not happen uniformly to all terminals. This is so because the method schedules packet transmissions with no regard of their reception quality. Instead, most of the loss happens to a group of terminals situated at "bad" locations (e.g., at the boundary of sectors/cells and unfavorable shadow fading). Given that terminal positions are fixed in the networks under consideration, this represents a major shortcoming. The ESRA method avoids that as it schedules packet transmissions according to the reception quality at the receiving terminals. 


\subsection{Uplink Performance for the ESRA Method}

As a natural choice, the same BS selected by a terminal for the downlink is also chosen for the uplink. Various terminals are scheduled to transmit uplink according to the same staggered order in Figure 3. As discussed earlier, the notion of terminal classification does not apply to the uplink. Instead, we use the traffic class to indicate the degree of concurrent transmissions. For example, terminals in sector $i$ are scheduled to transmit class 1 traffic in mini-frame 1 of subframe $i$ for all $i=1$ to 6 . For sending class 2 traffic, terminals in sector $i$ are scheduled to transmit in mini-frame 2 of subframe $i$ and $(i+3) \bmod 6$ for $i=1$ to 6 . Of course, the scheduling mechanism in the ESRA method arranges at most one terminal transmission in each sector at a time.

The uplink SIR performance for the ESRA method will not be acceptable when the strongest interfering terminal in each associated sector also transmits according to the staggered order. Table 2 shows that the lower and upper bound of the uplink SIR when such happens. It is clear from the Table that even for class 1 traffic (i.e., only one transmission in a cell at a time, but with the strongest interfering terminal in the corresponding sector also transmitting in all other cells), the lower bound of $9.85 \mathrm{~dB}$ is simply not adequate. The SIR performance degrades rapidly for other traffic classes with additional concurrent transmissions. Nevertheless, these results are too pessimistic because the strongest interfering terminals do not always transmit.

Instead, as in the actual operating environment, each uplink transmission experiences various degrees of interference, depending on the locations and fading conditions of other transmitting terminals. To quantify the uplink performance, we thus obtained the SIR distribution by evaluating $10^{5}$ random combinations of transmitting terminals, one in each associated sector according to the staggered order. Figure 12 and 13 show the SIR distribution with selected power control schemes for traffic class 1 and 2, respectively. As depicted in both figures, the FPC performs better than other power control schemes in the range of SIR between 10 to $15 \mathrm{~dB}$. Specifically, when the FPC is in use, the packet loss probability for an SIR threshold of $15 \mathrm{~dB}$ for class 1 traffic is $5 \times 10^{-4}$ in Figure 12, which is adequate for most real-time applications. The SPC and no power control perform poorly when the SIR is below $19 \mathrm{~dB}$.

Figure 13 shows the SIR distribution for class 2 traffic. Again, it is observed that the FPC performs better than the SPC when the SIR lies below 20dB. For a SIR detection threshold of 12 to $15 \mathrm{~dB}$, about $1 \%$ to $9 \%$ of packets cannot be received successfully under the FPC. This figure may be acceptable for applications with no stringent delay requirements. As a result, terminals can transmit their traffic as class 1 or 2 , depending on the quality requirements of the applications. 
It is worth pointing out that results in Figure 12 and 13 are the worst-case results because the SIR distributions were obtained by changing only the combinations of transmitting terminals, but each associated sector according to the staggered order always schedules one of its terminals for transmission. If the traffic load is low, less terminals transmit, thus improving the SIR performance as interference decreases.

It is also important to note that the distribution curves for the FPC and SPC cross over each other at about 20dB in Figure 12 and 13. Because of the large probability mass above the cross-over point, the average SIR for the SPC in fact is higher than that for the FPC. This is consistent with the optimality property of the SPC proven in [HWJ97]. However, as our objective is to maximize the SIR to support a given packet loss probability of less than a few percent (i.e., tail distribution), the FPC performs better than the SPC does, as shown in the figures. It is so because the FPC boosts the signal strength for a small fraction of combinations of transmitting terminals to meet the loss probability requirement, although the FPC in general causes more interference, thus yielding SIR lower than the SPC does on average. For class 2 traffic in Figure 13, the performance difference between the FPC and SPC for the SIR less than $20 \mathrm{~dB}$ is more drastic than that in Figure 12. This is because class 2 has concurrent transmissions within the same cell and, as shown above, the FPC is optimal to manage the intra-cell interference.

In terms of throughput, for class 1 traffic on the uplink, the ESRA throughput per sector is $16.67 \%$ as terminals in each sector $i$ can transmit in time subframe $i$ in the staggered order (i.e., one of the six subframes). In light of highly asymmetrical bandwidth consumption between downlink and uplink (with a ratio exceeding of 10 to 1 ) for data services such as for Internet applications [M97], such throughput adequately matches the downlink throughput of $36.10 \%$ in the ESRA method for the above parameter setting (which corresponds to the throughput result in Figure 5 with BS antenna beamwidth of $50^{\circ}$ ). For other applications with balanced bandwidth consumption, the uplink throughput can be improved by transmitting non-real-time traffic as class 2 and using the FPC to improve the SIR while meeting the packet loss probability requirements. Alternatively, to provide a balanced downlink and uplink bandwidth despite of their throughput difference, we can choose the relative size of their time frames in the TDD or assign different amount of frequencies to them in the FDD approach accordingly.

\subsection{Comparison of Uplink Performance With CDPA and SRA Method}

We remark that although the uplink protocol in both the ESRA and SRA method operate in a similar way, the key differences between them are: 1) the ESRA uses the FPC for the uplink, while the SRA method assumes no power control, and 2) the ESRA selects the mini-frame sizes according to the expected traffic load of different classes, which are defined according to the number of concurrent 
transmissions in each cell.

As for the CDPA uplink protocol, at most one terminal in each sector is scheduled to transmit in a time slot. To conform with the traffic classes define above, multiple sectors in each cell may have concurrent transmissions in the CDPA method. To schedule class $i$ traffic under the CDPA scheme in the sectorized-cell setting described in Section 6.1, our model first randomly selects $i$ sectors in each cell. Then one random terminal in each of the sectors is chosen for uplink transmission.

Figure 14 and 15 present a comparison of uplink SIR performance among the ESRA, SRA and CDPA schemes for class traffic 1 and 2, respectively. In both figures, the high SIR percentiles (e.g., 99\% or $90 \%$ ) for the SRA method are higher than those for the CDPA method. This is so because the staggered order helps the SRA method avoid major interference. As explained above, the FPC in the ESRA method further improves the tail probability of the SIR, when compared with the SRA method.

\subsection{Quality of Service (QoS)}

As discussed in [FHLQS98], the SRA method can be used in conjunction with a control mechanism to limit the degree of concurrent transmissions for enhancing the throughput and success probability of packet transmission. Essentially, the ESRA method by use of mini-frames applies various control limits to control the degree of concurrent transmissions, depending on the reception quality at the terminal locations. By the terminal classification and the scheduling algorithm, downlink transmissions for all terminal classes in the ESRA method will be successfully received because they meet a specific SIR threshold. As for the uplink, the packet loss probability can be kept satisfactorily low with the FPC for typical modulation and equalization schemes. Thus, the ESRA performance is stable in a sense that its throughput and successful packet transmission do not deteriorate when offered with an excessive amount of traffic. (Of course, the queueing delay will become unbounded for excessive traffic load.) For these reasons, the ESRA method will be adequate even for real-time traffic such as voice and video services. In addition, success probability of one for downlink transmission and adequately low packet loss probability for uplink, can help simplify the call admission control and traffic management to ensure the desirable level of QoS.

Of course, the ESRA downlink performance depends on the correct categorization of terminals. As the quality of a radio path between any pair of BS and terminal may change over time, perhaps due to seasonal fluctuation or man-made objects, it is important to monitor the reception quality periodically by measurements and statistics collection. When needed, a terminal can be re-classified. To handle temporary fluctuation of a radio link, the ESRA method can use the upgraded sharing approach to retransmit packets on the downlink (i.e., to make sure that class- $c$ packets are re-transmitted in mini-frame $k$ 
with $k<c$ ) that are not received properly in the first attempt. As discussed above, when packet transmissions fail repeatedly for a terminal, it can be re-classified dynamically.

\section{CONCLUSION}

We have proposed the ESRA method for radio-resource allocation for both downlink and uplink in fixed broadband wireless networks, which is based on the SRA algorithm proposed earlier with consideration of reception quality at terminal locations. The new method maximizes the degree of concurrent packet transmissions up to an extent tolerable by the receiving terminals, while it is capable of of avoiding major intra- and inter-cell, co-channel interference in networks where a given frequency band is re-used in every sector of every cell.

For reasonable radio environment, practical antenna patterns and choices of system parameters, the ESRA method provides $98.64 \%$ coverage, and yields a maximum downlink throughput of $36.10 \%$ per sector with success probability of one for packet transmission in terms of meeting a $15 \mathrm{~dB}$ SIR threshold. As for the uplink, the throughput can reach $16.7 \%$ per sector with a packet loss probability of less than

$5 \times 10^{-4}$, when the full power control is used. The ESRA performance is compared favorably to the CDPA and SRA method. The high ESRA throughput translates into a very large network capacity. In addition to data services, the high quality of service reveals that the new method can be used to support real-time traffic such as voice and video. Therefore, we believe that the new approach represents an important step forward in providing broadband, multi-media services in fixed wireless networks.

\section{ACKNOWLEDGMENTS}

Special thanks are due to K. Chawla and X. Qiu for sharing their ideas and results with us that motivated this work. We thank N.K. Shankaranarayanan for his discussion and help in simulation. Thanks are also due to J.H. Winters and P.S. Henry for their discussion and comments.

\section{REFERENCES}

[AEK96] E. Ayanoglu, K.Y. Eng and M.J. Karol, "Wireless ATM: Limits, Challenges, and Proposals," IEEE Personal Communications, pp.18-34, August 1996.

[B96] Special Issue on Two-Way Broadband Access Networks, Bell Labs Technical Journal, Vol.1, No.1, Summer 1996.

[BFZA96] F. Borgonovo, L. Fratta, M. Zorzi and A. Acampora, "Capture Division Packet Access: A New Cellular Access Architecture for Future PCNs," IEEE Communications Magazine, pp.154-162, 
Sept. 1996,

[BMSS96] C. Bisdikian, K. Maruyama, D.I. Seidman and D.N. Serpanos, "Cable Access Beyond the Hype: On Residential Broadband Data Services Over HFC Networks," IEEE Communications Magazine, pp.128-135, Nov. 1996.

[BZFTB96] F. Borgonovo, M. Zorzi, L. Fratta, V. Trecordi and G. Bianchi, "Capture-Division Packet Access for Wireless Personal Communications," IEEE J. Select. Areas in Commun., Vol.14, No.4, pp.609-622, May 1996.

[FHLQS98] T.K. Fong, P.S. Henry, K.K. Leung, X. Qiu and N.K. Shankaranarayanan, "Radio Resource Allocation in Fixed Broadband Wireless Networks," IEEE Trans. on Commun., Vol.46, No.6, pp.806-818, June 1998.

[FM93] G.J. Foschini and Z. Miljanic, "A Simple Distributed Autonomous Power Control Algorithm and Its Convergence," IEEE Trans. on Veh. Tech., Vol.42, No.4, pp.57-62, Nov. 1993.

[GJ79] M.R. Garey and D.S. Johnson, Computers and Intractability: A Guide to the Theory of NPCompleteness, W.H. Freeman and Company, San Francisco, 1979.

[HF97] M. Humphrey and J. Freeman, "How xDSL Supports Broadband Services to the Home," IEEE Network, Jan./Feb. 1997, pp.14-23.

[HWJ97] Z.J. Haas, J.H. Winters and D.S. Johnson, "Simulation Results of the Capacity of Cellular Systems," IEEE Trans. on Vech. Tech., Vol.46, No.4, pp.805-817, Nov. 1997.

[197] Special Issue on Broadband Services Over Residential Networks, IEEE Network, Vol.11, No.1, Jan./Feb. 1997.

[KN96] I. Katzela and M. Naghshineh, "Channel Assignment Schemes for Cellular Mobile Telecommunication Systems: A Comprehensive Survey," IEEE Personal Communications, pp.10-31, June 1996.

[KW97] N. Kahale and P.E. Wright, "Dynamic Global Packet Routing in Wireless Networks," Proc. of IEEE INFOCOM’97, Kobe, Japan, April 1997, pp.1416-1423.

[L89] W.C.Y. Lee, Mobile Cellular Telecommunications Systems, McGraw-Hill, New York, 1989.

[M96] K. Maxwell, "Asymmetrical Digital Subscriber Line: Interim Technology for the Next Forty Years," IEEE Communications Magazine, Vo.34, No.10, Oct. 1996, pp.100-106. 
[M97] B.A. Mah, "An Empirical Model of HTTP Network Traffic," Proc. of IEEE INFOCOM'97, Kobe, Japan, April 1997, pp.593-602.

[QC97] X. Qiu and K. Chawla, "Resource Assignment in a Fixed Wireless System," IEEE Commun. Letters, Vol. 1, No.4, pp.108-110, July 1997.

[R96] T.S. Rappaport, Wireless Communications: Principles and Practice, IEEE Press and Prentice Hall PTR, New York, 1996.

[SK98] D. Sobirk and J.M. Karlsson, "A Survey of Wireless ATM MAC Protocols," Performance and Management of Complex Communication Networks, T. Hasegawa, H. Takagi, Y. Takahashi (Ed.), Chapman \& Hall, New York (1998).

[W93] J.F. Whitehead, "Signal-level-based Dynamic Power Control for Co-channel Interference Management, Proc. of IEEE Vech. Tech. Conf., Secaucus, NJ, May 1993, pp.499-502.

[W96] J.F. Whitehead, private communications, 1996.

[Z92a] J. Zander, "Performance of Optimum Transmitter Power Control in Cellular Radio Systems" IEEE Trans. on Veh. Tech., Vol.41, No.1, pp.57-62.

[Z92b] J. Zander, "Distributed Cochannel Interference Control in Cellular Radio Systems," IEEE Trans. on Veh. Tech., Vol.41, No.3, pp.305-311, Aug. 1992.

[Z97] M. Zorzi, "On the Analytical Computation of the Interference Statistics with Applications to the Performance Evaluation of Mobile Radio Systems," IEEE Trans. on Commun., Vol.45, No.1, Jan. 1997, pp.103-109.

[ZTL96] G.I. Zysman, R. Thorkildsen and D.Y. Lee, "Two-Way Wireless Broadband Access," Bell Labs Technical Journal, Summer 1996, pp.115-129. 

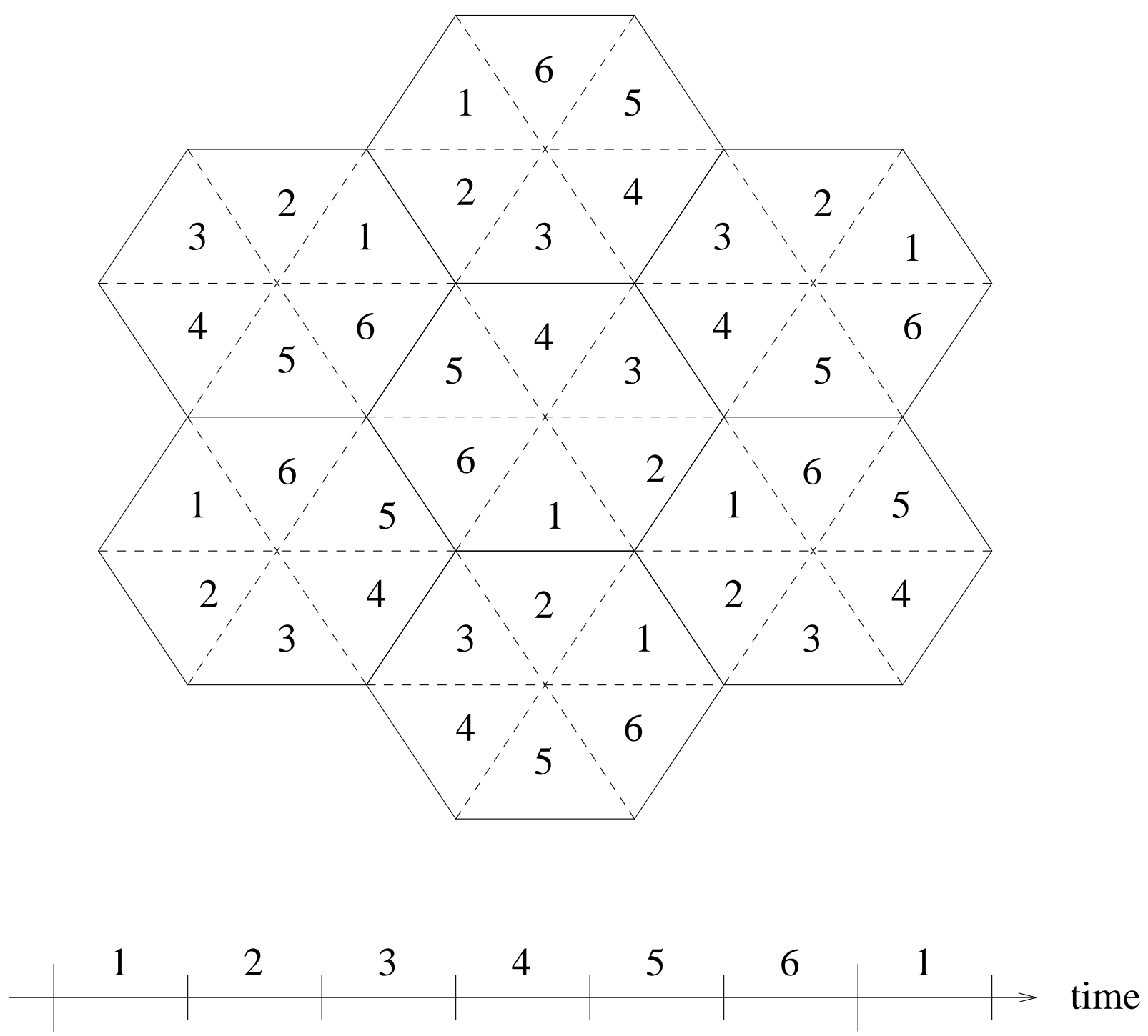

Figure 1. Cell Layout and Frame Structure 
Time Subframes

\begin{tabular}{|c|c|c|c|c|c|c|c|}
\hline & 1 & 2 & 3 & 4 & 5 & 6 & time \\
\hline \multicolumn{8}{|c|}{ Sector } \\
\hline 1 & $\mathrm{a}$ & $\mathrm{e}$ & $d$ & b & $\mathrm{c}$ & $\mathrm{f}$ & \\
\hline 2 & $\mathrm{f}$ & $\mathrm{a}$ & e & d & b & $c$ & \\
\hline 3 & $\mathrm{c}$ & $\mathrm{f}$ & $\mathrm{a}$ & e & d & $\mathrm{b}$ & \\
\hline 4 & b & $\mathrm{c}$ & $\mathrm{f}$ & $\mathrm{a}$ & e & $d$ & \\
\hline 5 & $d$ & b & $\mathrm{c}$ & $\mathrm{f}$ & $\mathrm{a}$ & e & \\
\hline 6 & e & $d$ & b & $c$ & $\mathrm{f}$ & $\mathrm{a}$ & \\
\hline
\end{tabular}

Figure 2. Order of Slot Assignment for the SRA Method 


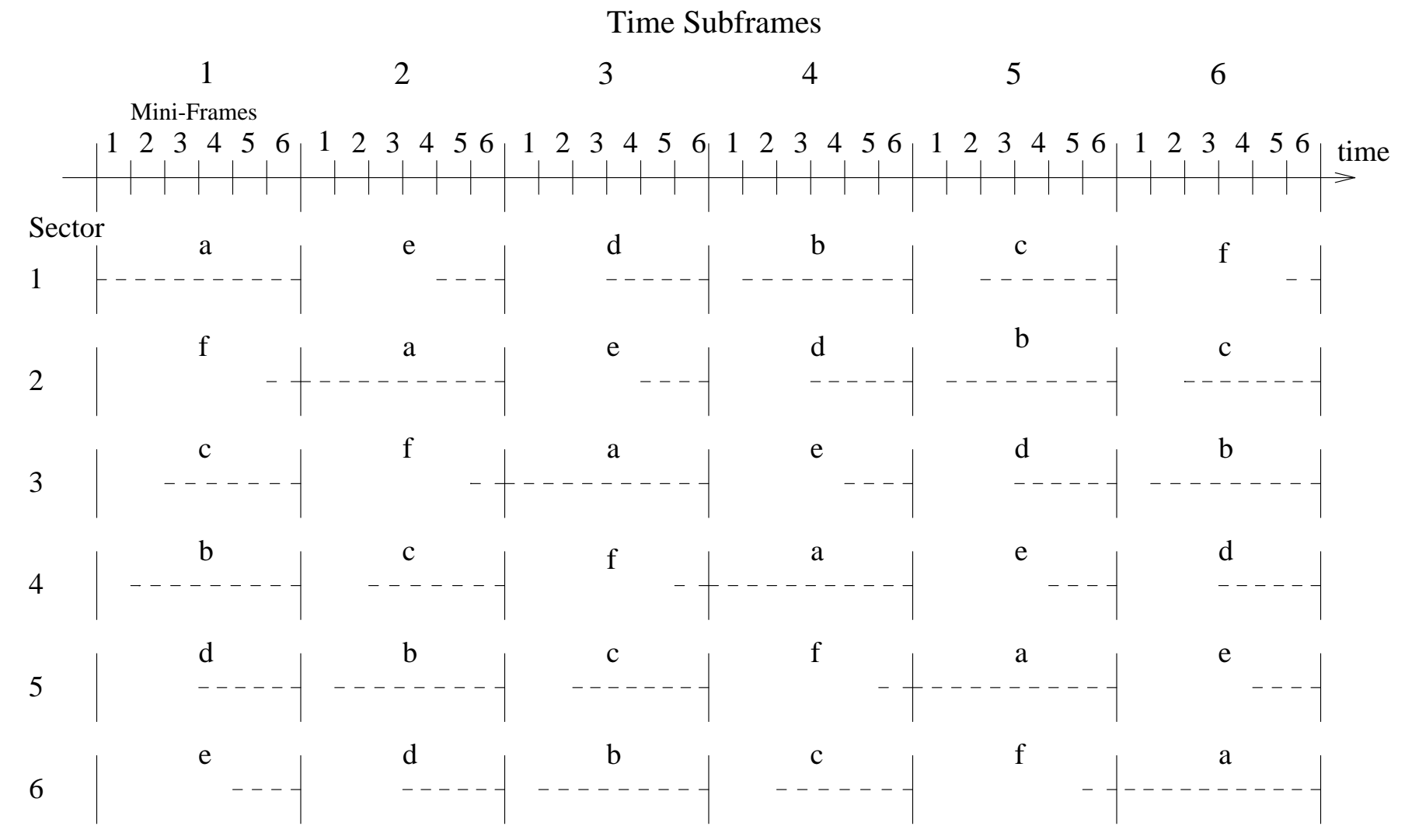

Figure 3. Use Order of Subframes and Mini-Frames in the ESRA Method 


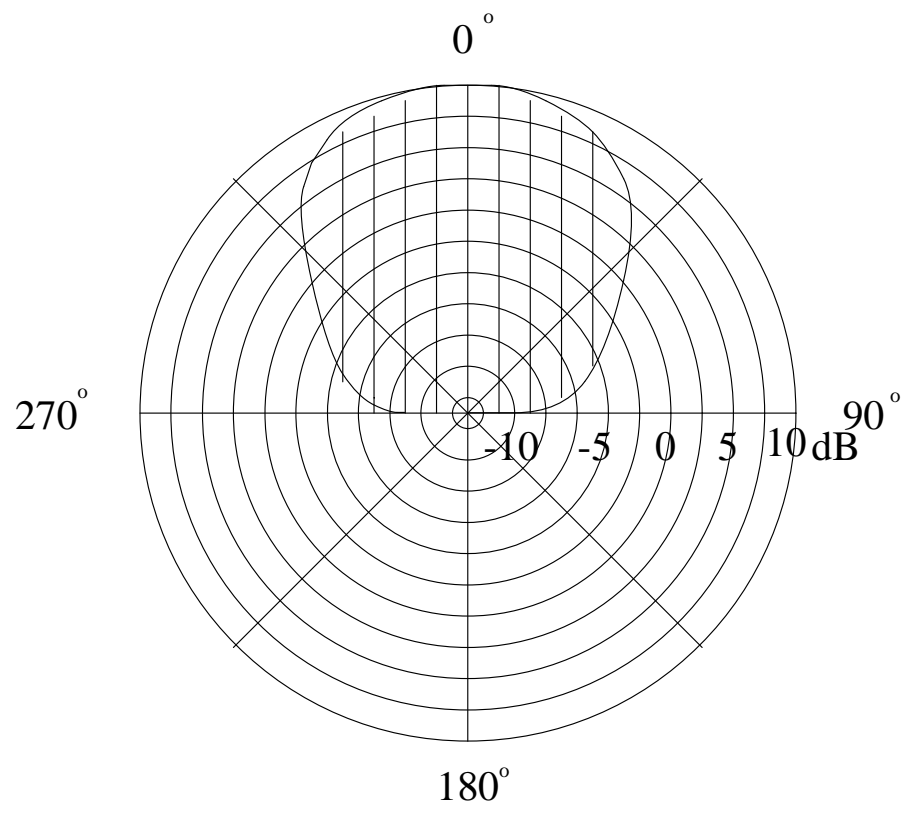

Figure 4: A Typical Antenna Pattern with FTB Ratio of $25 \mathrm{~dB}$ and and Beamwidth of 60 degrees 


\begin{tabular}{|c|c|c|}
\hline Terminal Class & Without BS Selection & With BS Selection \\
\hline 1 & 0.1146 & 0.0813 \\
2 & 0.5250 & 0.6375 \\
3 & 0.0333 & 0.0479 \\
4 & 0.0115 & 0.0208 \\
5 & 0.0219 & 0.0292 \\
6 & 0.1531 & 0.1698 \\
\hline Coverage & 0.8594 & 0.9864 \\
Throughput & 0.3402 & 0.3610 \\
\hline
\end{tabular}

Table 1. Fraction of Terminals in Various Classes for Downlink 
Figure 5. Impacts of BS Antenna Beamwidth on Downlink Performance

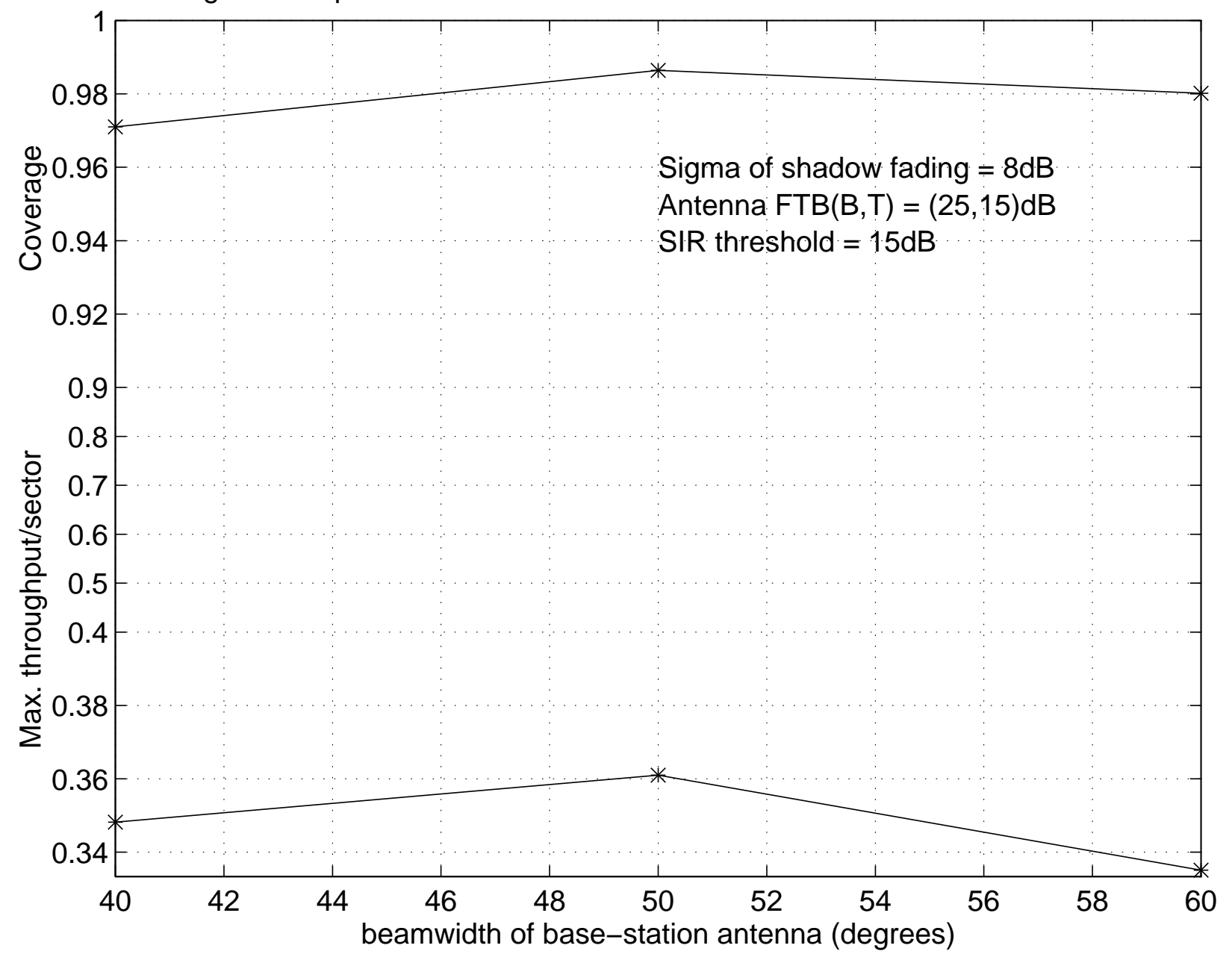


Figure 6. Impacts of FTB Ratio of BS Antenna on Downlink Performance

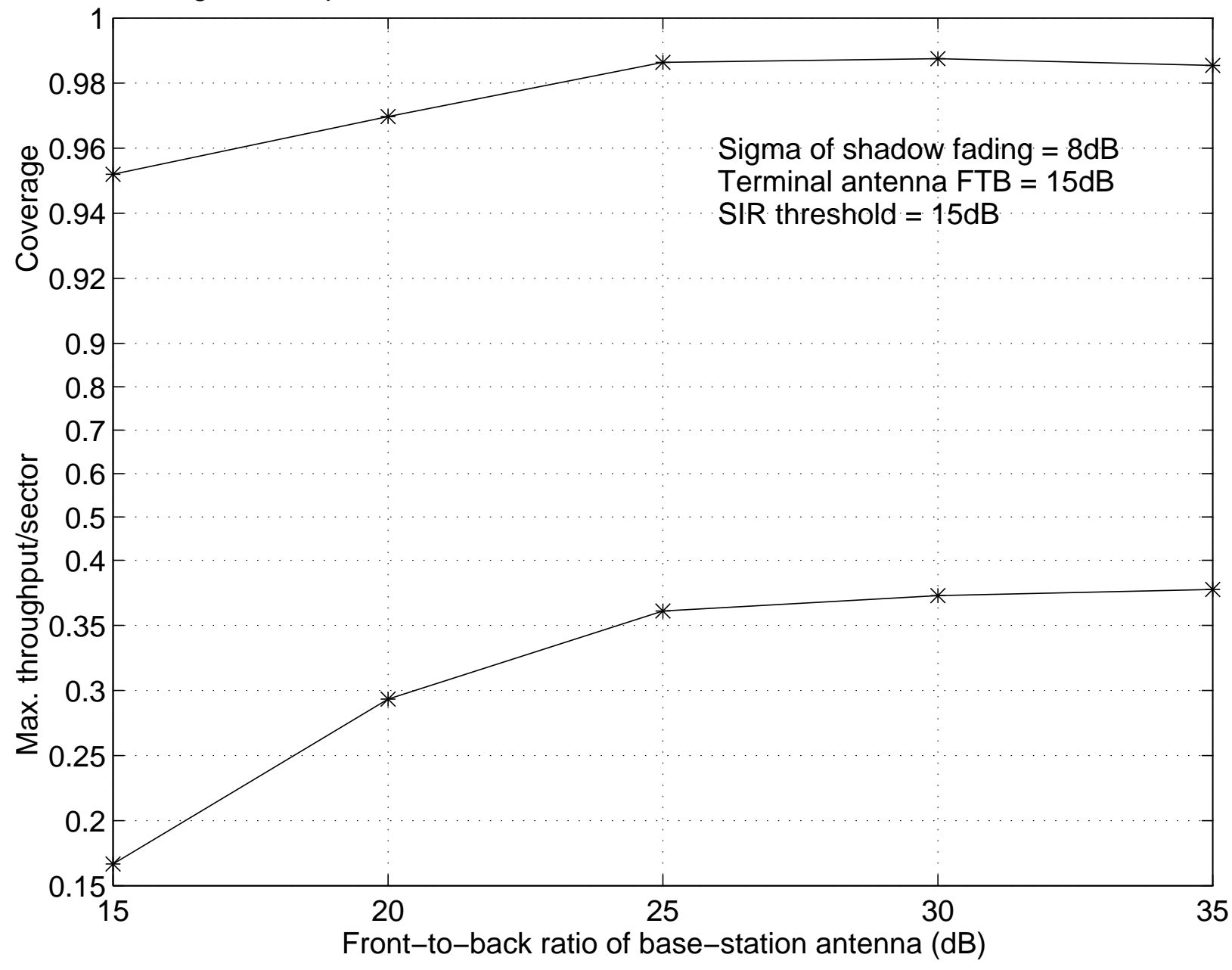


Figure 7. Impacts of FTB Ratio of Terminal Antenna on Downlink Performance

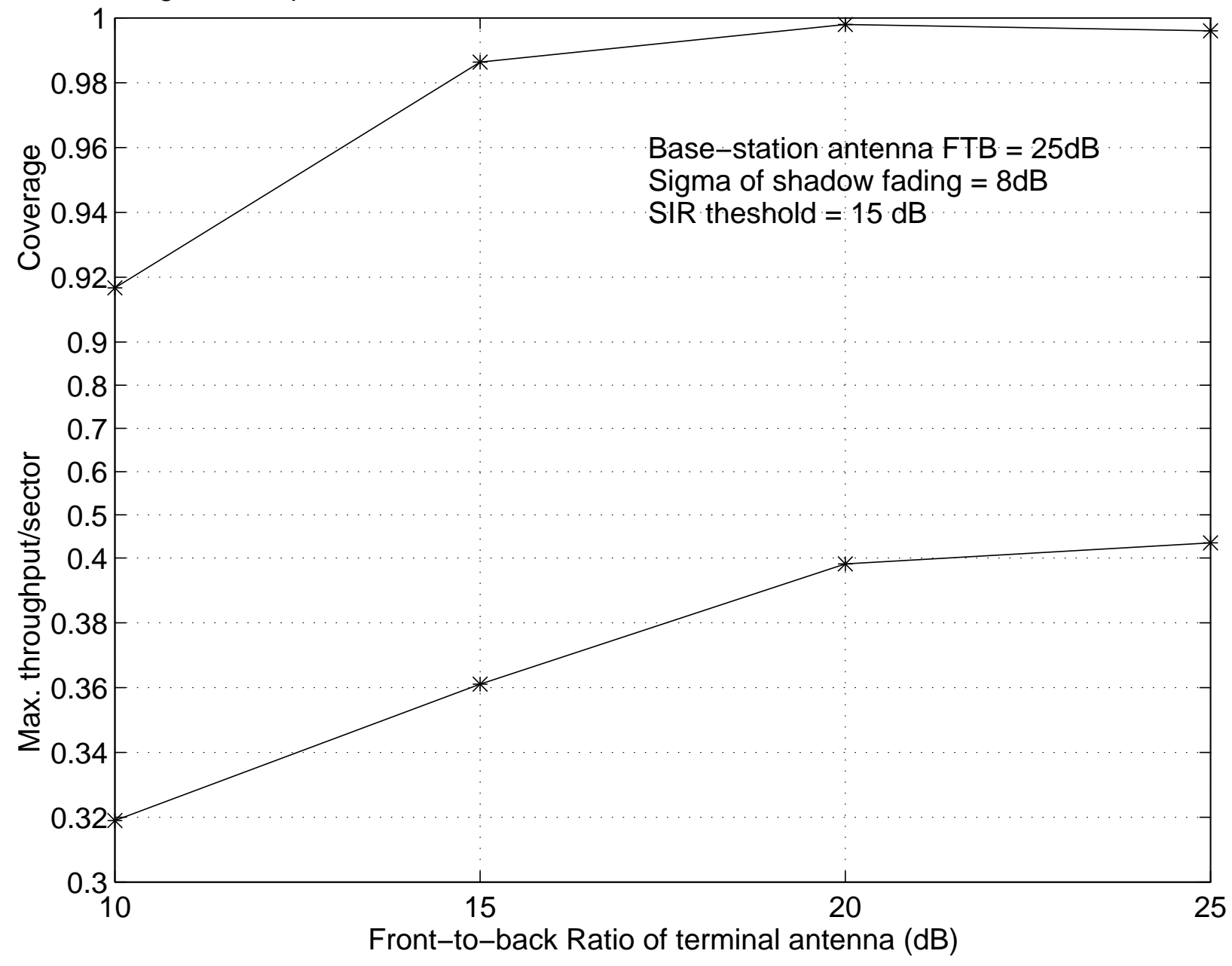


Figure 8. Throughput Comparison of the ESRA and CDPA Scheme

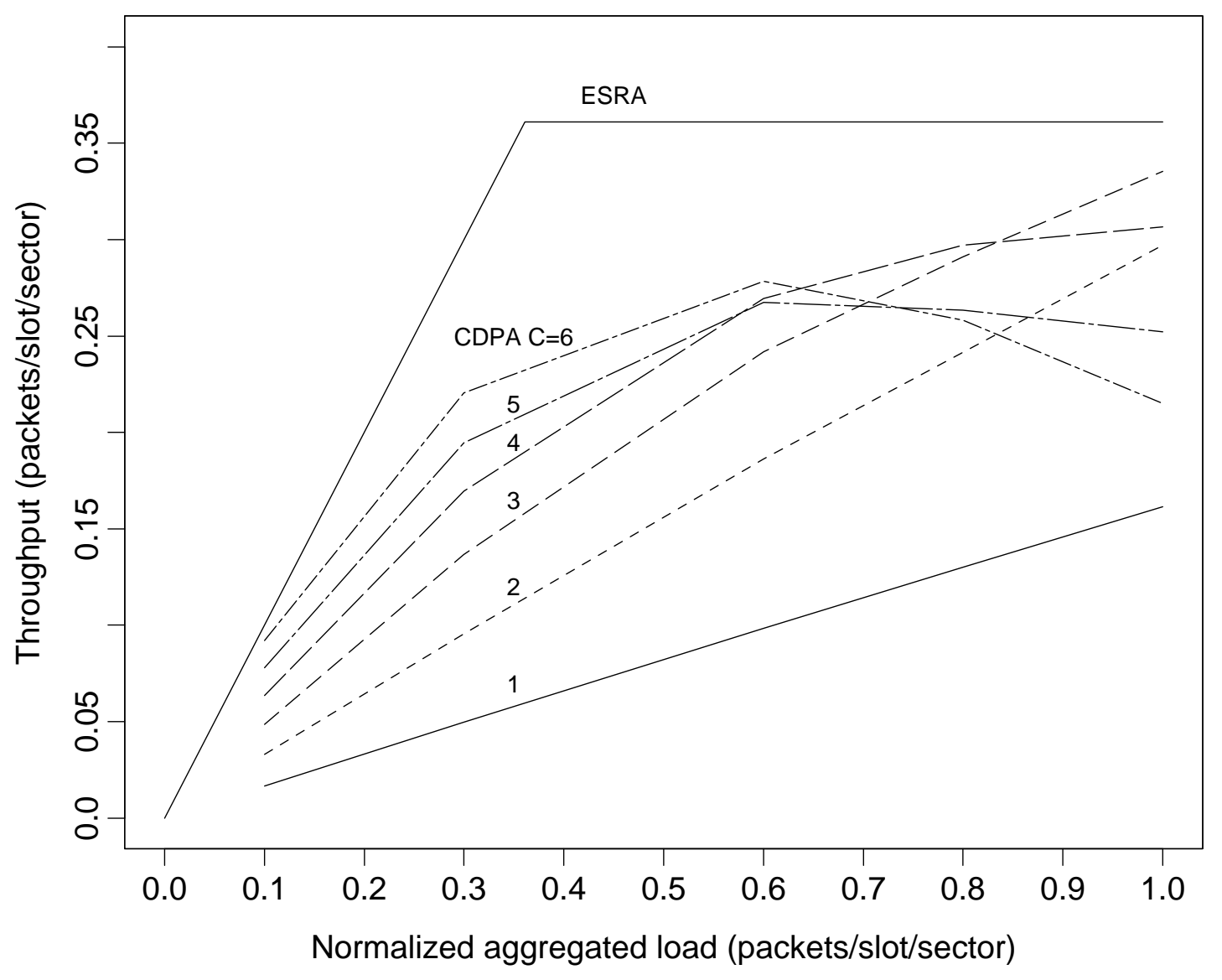


Figure 9. Comparison of Packet Success Probability Between the ESRA and CDPA Scheme

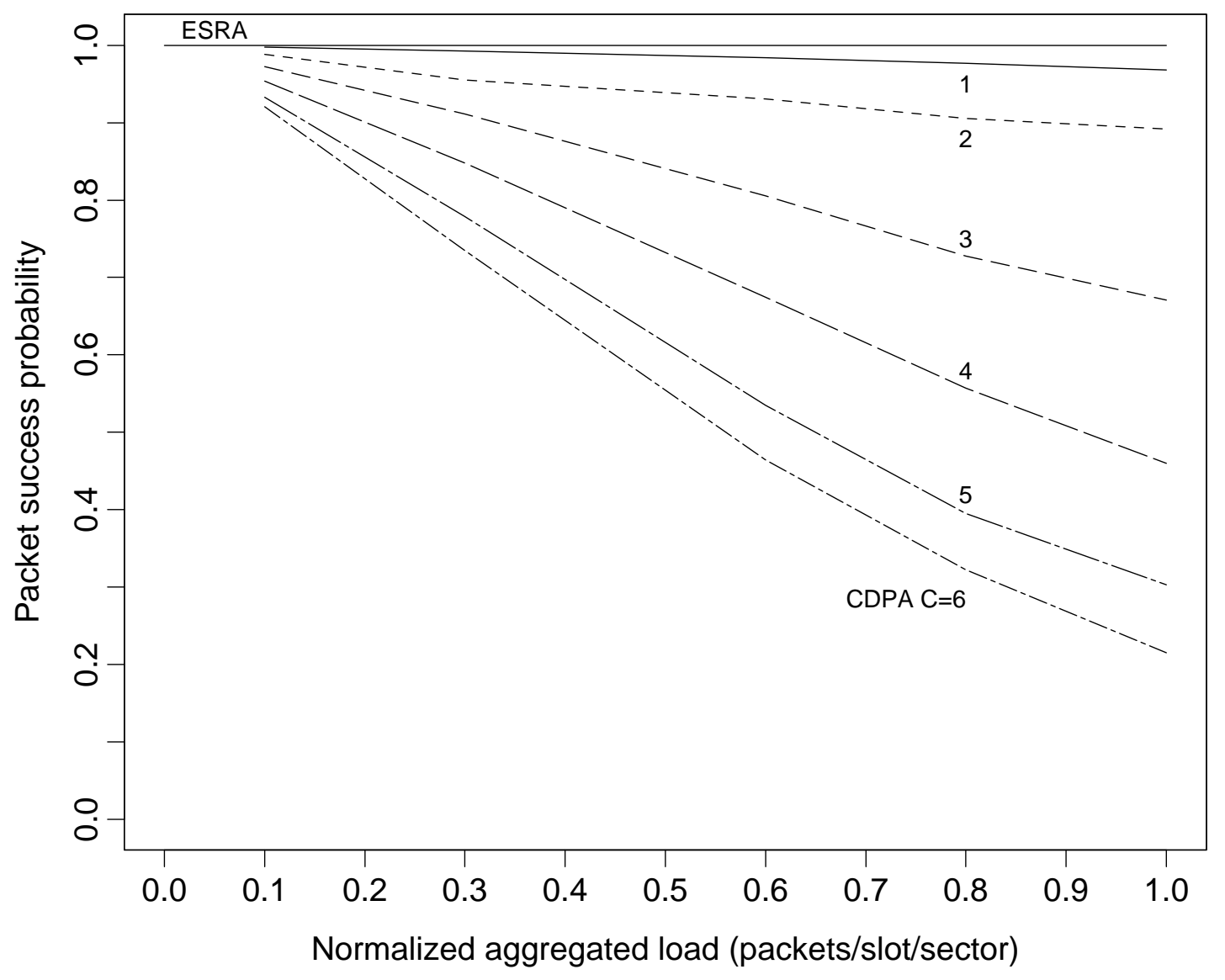


Figure 10. Throughput Comparison of the ESRA and SRA Method

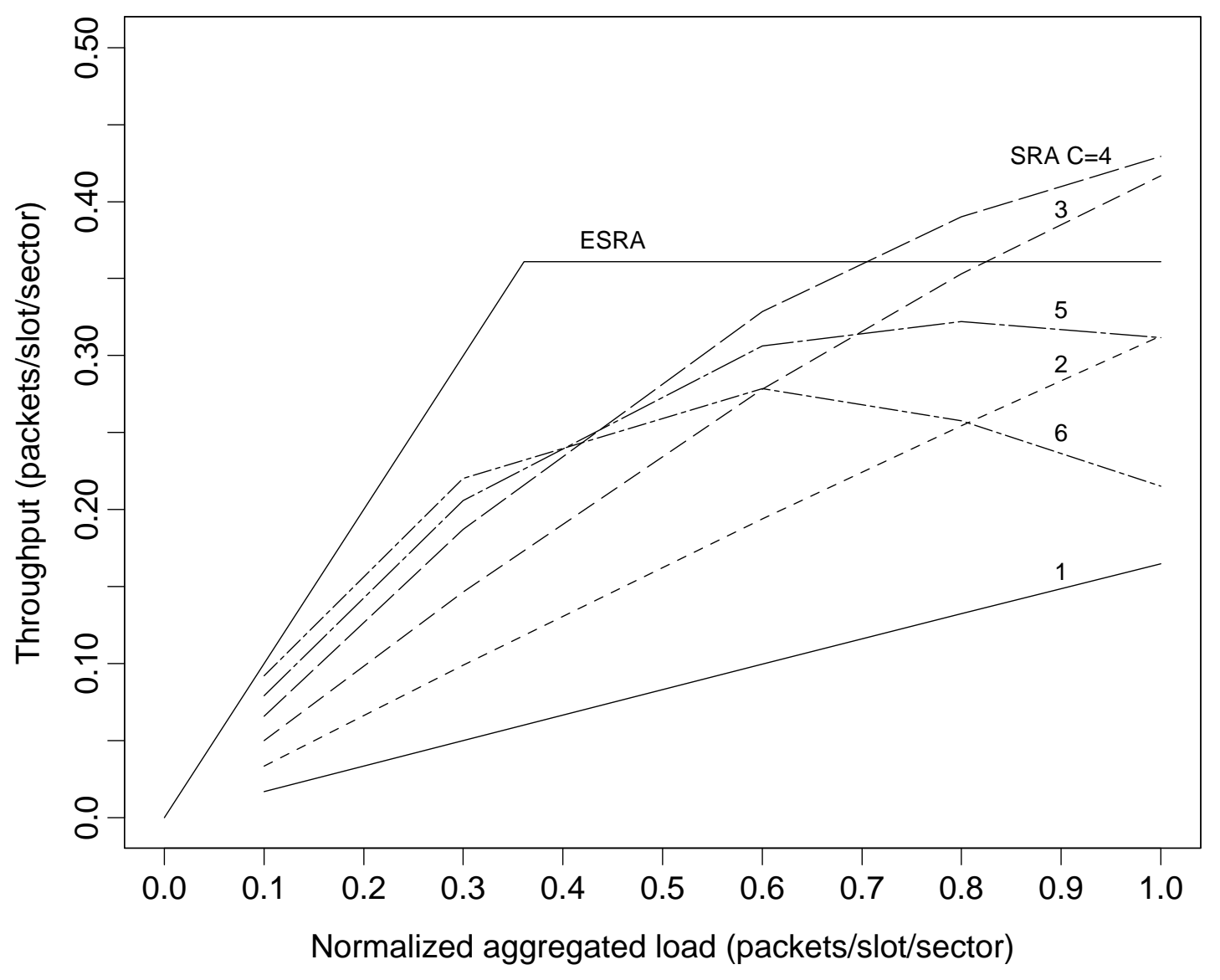


Figure 11. Comparison of Packet Success Probability Between the ESRA and SRA Method

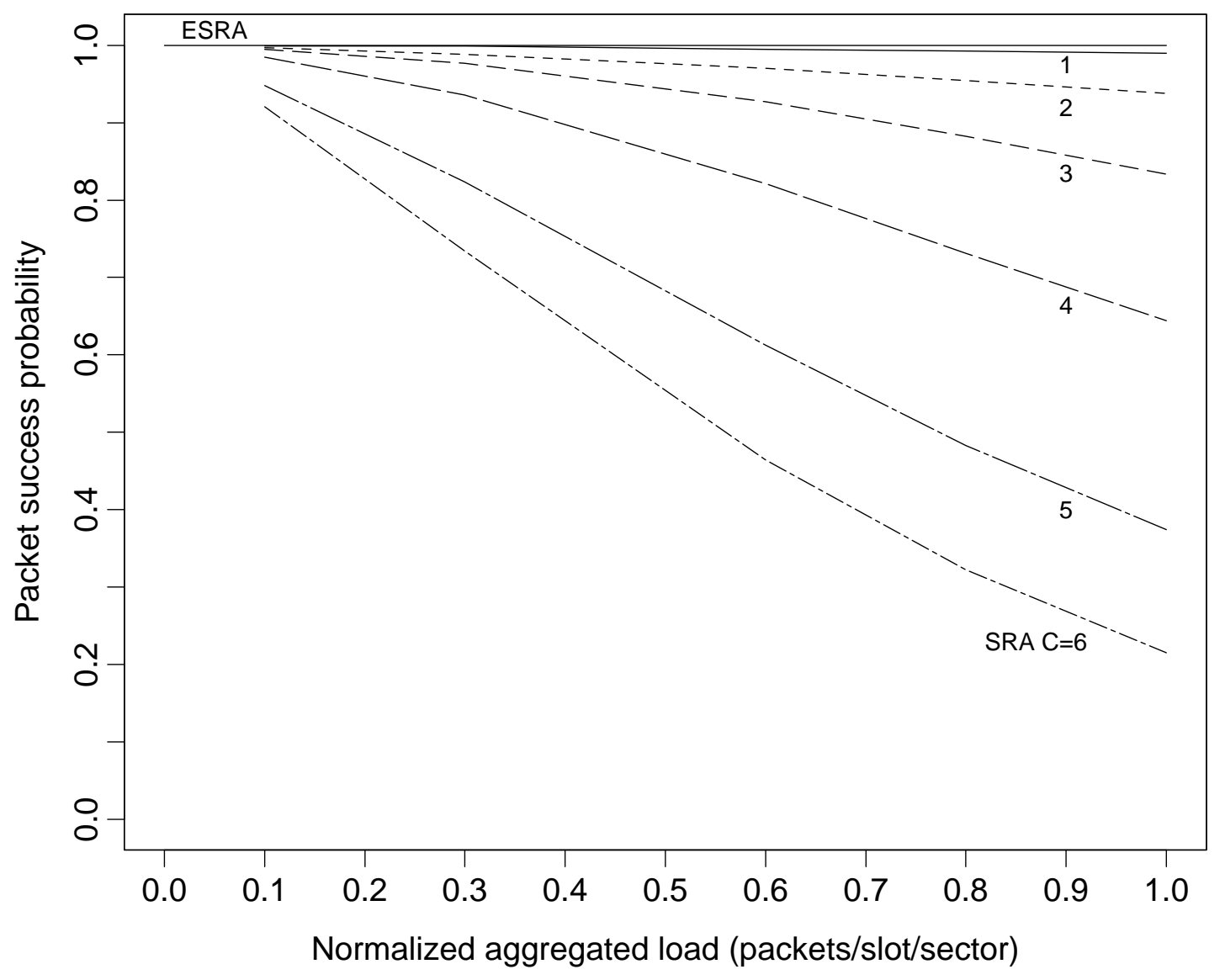




\begin{tabular}{|c|c|c|}
\hline \multirow{2}{*}{ Traffic Class } & \multicolumn{2}{|c|}{ Uplink SIR (dB) } \\
\cline { 2 - 3 } & Lower Bound & Upper Bound \\
\hline 1 & 9.85 & 16.19 \\
2 & 7.77 & 12.46 \\
3 & 0.66 & 4.18 \\
& & \\
4 & -1.7 & 2.14 \\
5 & -2.11 & 1.572 \\
6 & -2.3 & 1.2695 \\
\hline
\end{tabular}

\section{Table 2. Ranges of Uplink SIR With Strongest Interferers in Neighboring Cells}


Figure 12. Impacts of Power Control on Uplink Performance for Class 1 Traffic

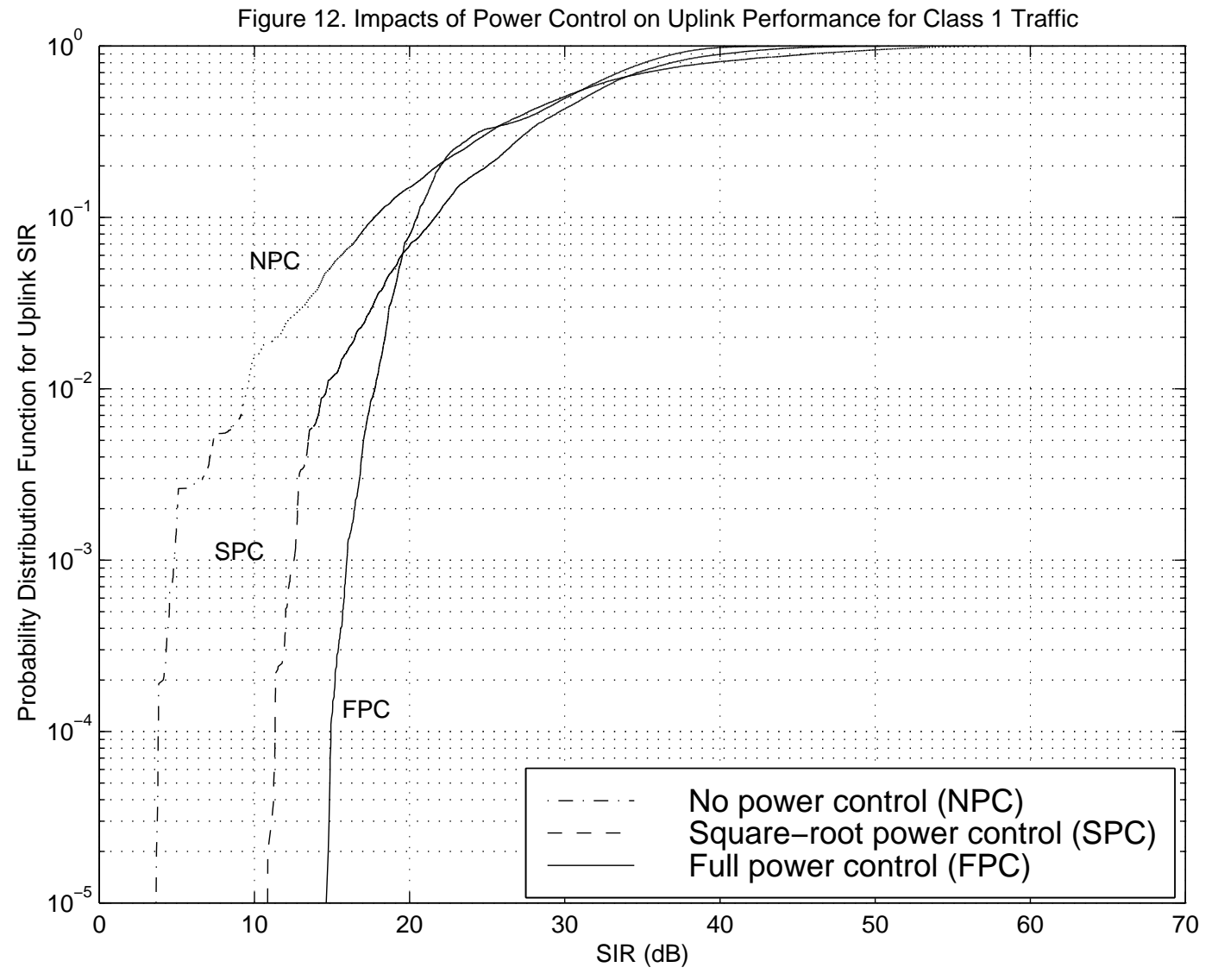


- 40 -

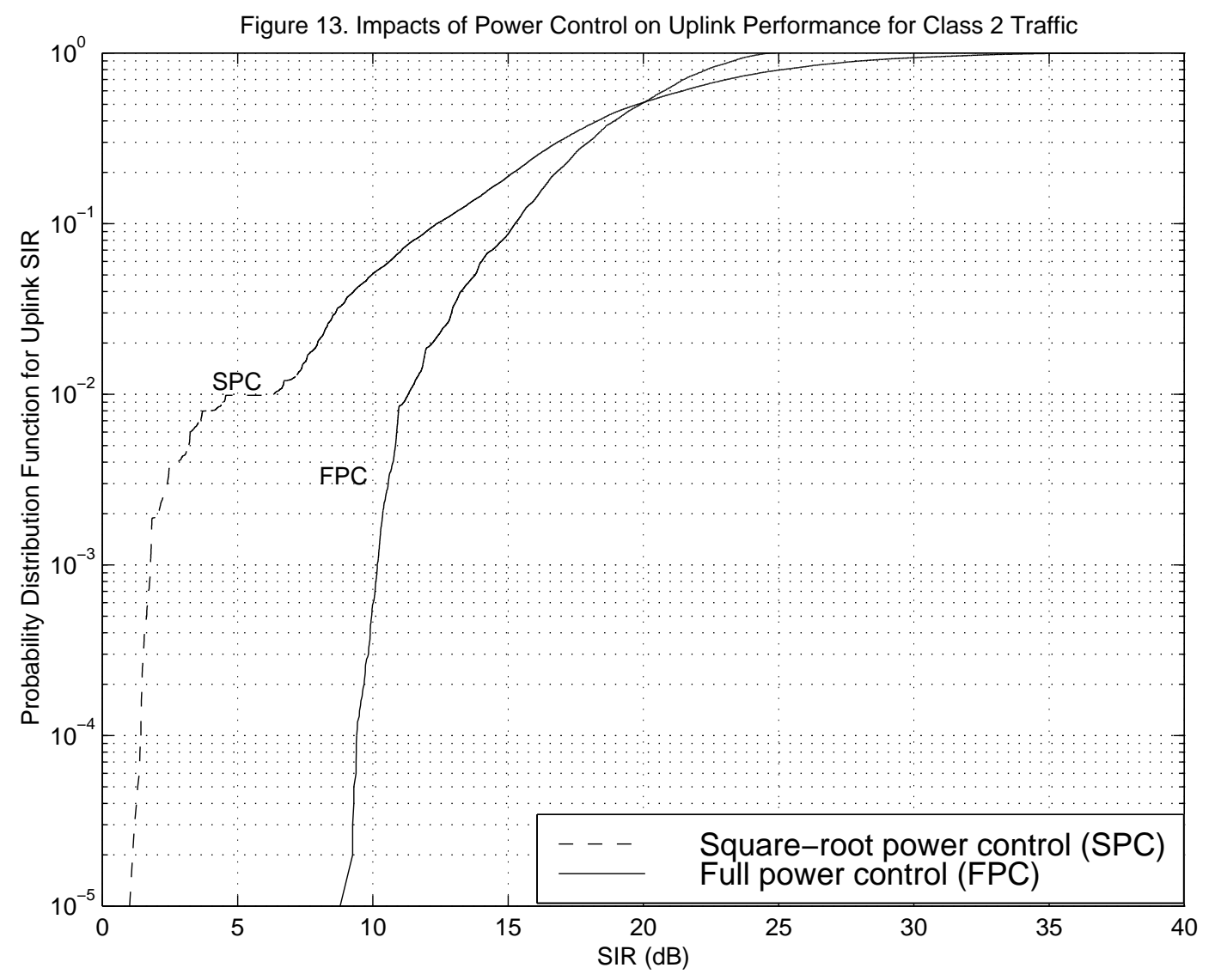


Figure 14. Comparison of Uplink Performance for Class 1 Traffic

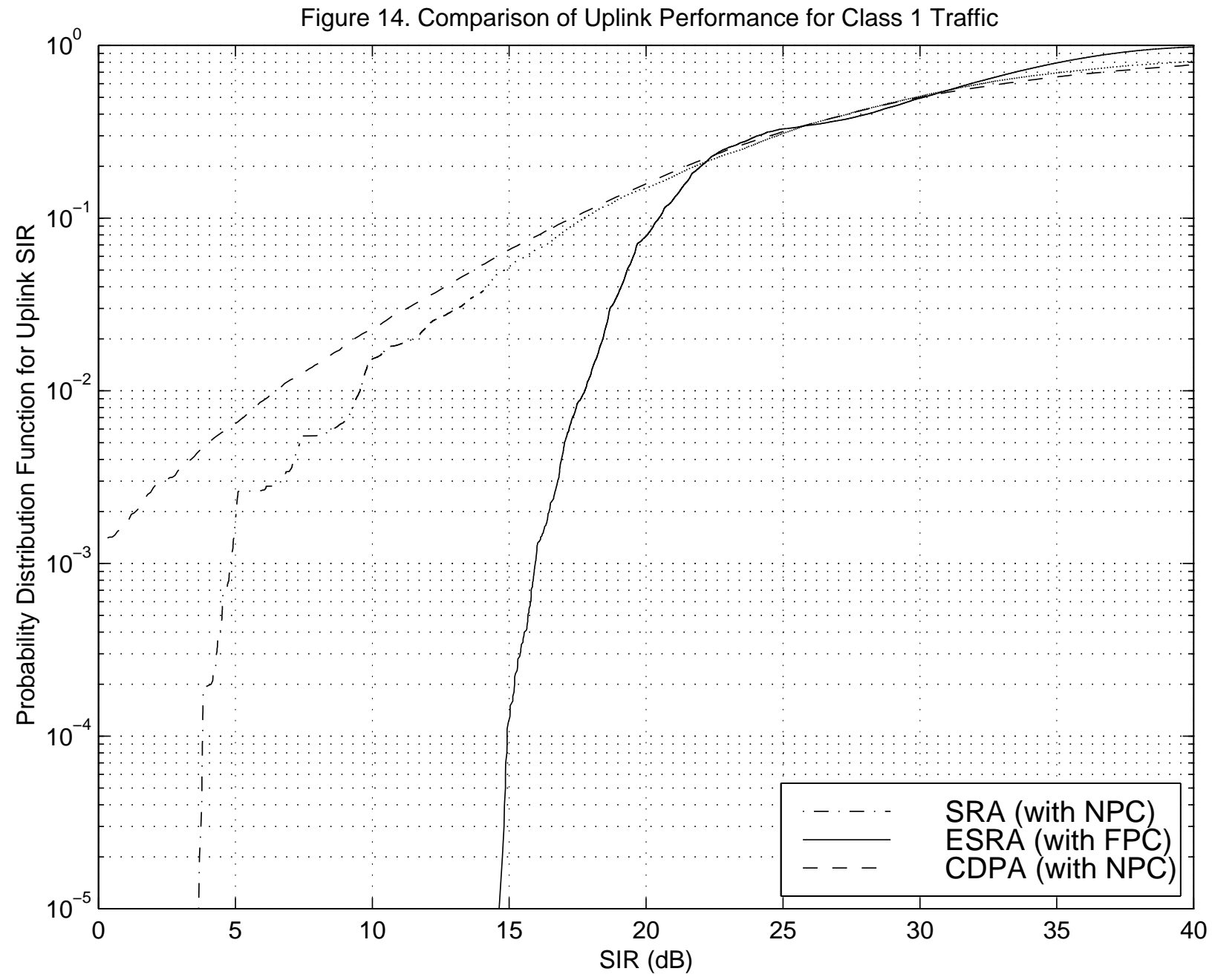


Figure 15. Comparison of Uplink Performance for Class 2 Traffic

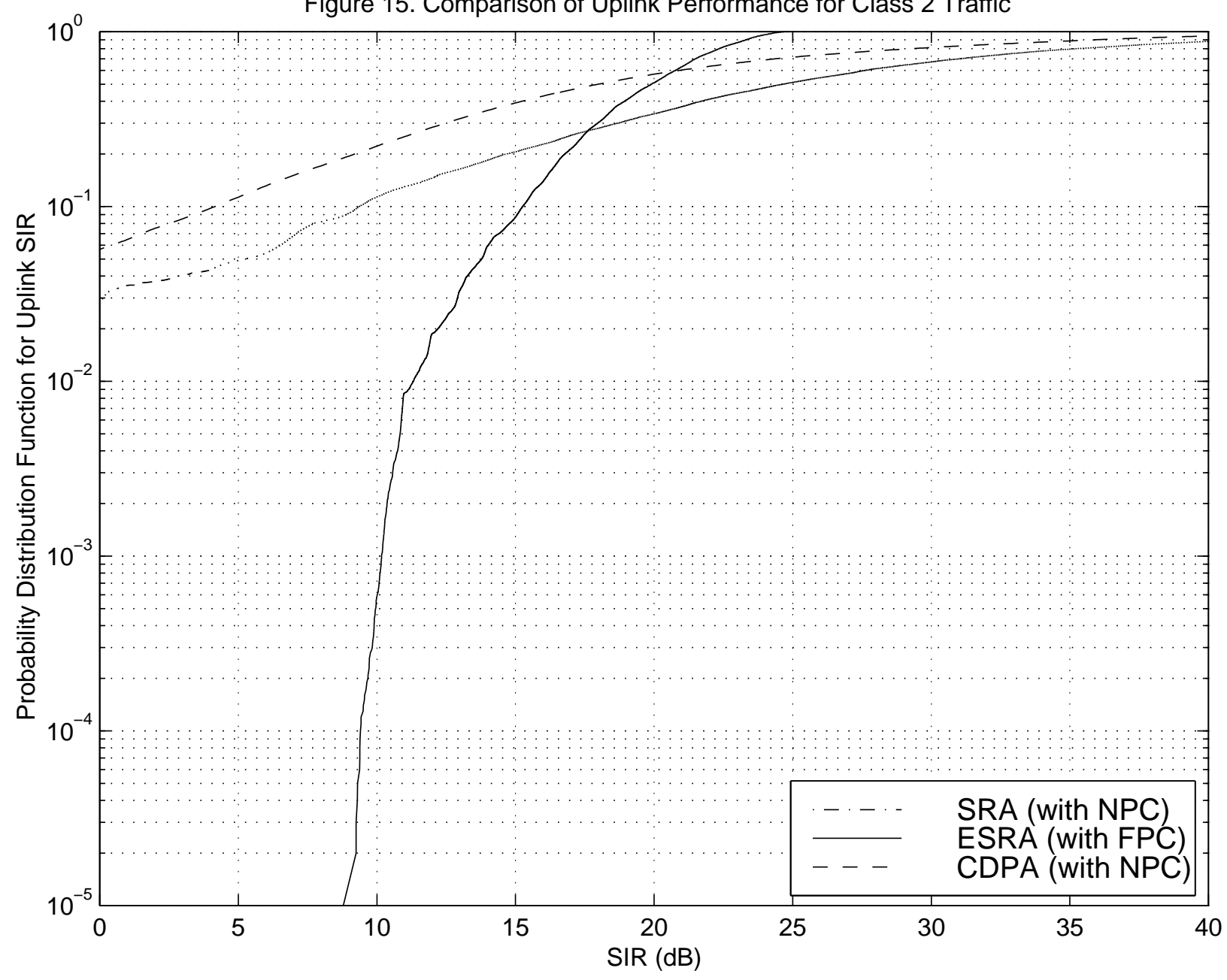

